\title{
Transgenic expression of Hyp-1 gene from Hypericum perforatum L. alters expression of defense-related genes and modulates recalcitrance to Agrobacterium tumefaciens
}

Weina Hou ${ }^{1}$, Rupesh Kumar Singh ${ }^{2}$, Pan Zhao ${ }^{3}$, Viviana Martins ${ }^{4}$, Emmanuel Aguilar ${ }^{5}$, Tomás Canto ${ }^{5}$, Francisco Tenllado ${ }^{5 *}$, Alberto Carlos Pires Dias ${ }^{1,4,6 *}$

${ }^{1}$ Centre for the Research and Technology of Agro-Environmental and Biological Sciences (CITAB), Department of Biology, University of Minho, 4710-057, Braga, Portugal

${ }^{2}$ Centro de Química de Vila Real (CQ-VR), UTAD, 5000-801, Vila Real, Portugal

${ }^{3}$ National Key Laboratory of Plant Genomics, Institute of Microbiology, Chinese Academy of

Sciences, Beijing, 100101, China

${ }^{4}$ Centre of Molecular and Environmental Biology (CBMA) , Department of Biology, University of Minho, 4710-057, Braga, Portugal

${ }^{5}$ Departamento de Biotecnología Microbiana y de Plantas , Centro de Investigaciones Biol ógicas, CSIC, Madrid, 28040, Spain

${ }^{6}$ Center of Biological Engineering (CEB), University of Minho, 4710-057, Braga, Portugal

*Corresponding authors: tenllado@cib.csic.es; albertocpdias66@gmail.com. Tfno: 34-91-8373112. Fax: 34-91-5360432

Declarations of interest: none

\section{Main Conclusion}

Phenolic oxidative coupling protein ( Hyp-l) isolated from Hypericum perforatum $\mathrm{L}$. was characterized as a defense gene involved into $H$. perforatum L. recalcitrance to Agrobacterium tumefaciens-mediated transformation. 


\begin{abstract}
Hypericum perforatum L. is a reservoir of high-value secondary metabolites of increasing interest to researchers and to the pharmaceutical industry. However, improving their production via genetic manipulation is a challenging task as $H$. perforatum L. is recalcitrant to Agrobacterium tumefaciensmediated transformation. Here, Phenolic oxidative coupling protein (Hyp-1), a pathogenesis-related (PR) class 10 family gene, was selected from a subtractive cDNA library from A.tumefaciens-treated H. perforatum L. suspension cells. The role of Hyp-1 in defense against A.tumefaciens was analyzed in transgenic Nicotiana tabacum and Lactuca sativa overexpressing Hyp-1, and in Catharanthus roseus silenced for its homologous Hyp-1 gene, CrIPR. Results showed that Agrobacterium-mediated expression efficiency greatly decreased in $H y p-1$ transgenic plants. However, silencing of $C r I P R$ induced $C r P R-5$ expression and decreased expression efficiency of Agrobacterium. The expression of core genes involved in several defense pathways was also analyzed in Hyp-l transgenic tobacco plants. Overexpression of Hyp-1 led to an ample down-regulation of key genes involved in auxin signaling, microRNA-based gene silencing, detoxification of reactive oxygen species phenylpropanoid pathway and PRs. Moreover, Hyp-1 was detected in the nucleus, plasma membrane and the cytoplasm of epidermal cells by confocal microscopy. Overall, our findings suggest $H y p-1$ modulates recalcitrance to A. tumefaciens-mediated transformation in H. perforatum L.
\end{abstract}

Keywords: H. perforatum L secondary metabolites, Agrobacterium tumefaciens-mediated expression, Hyp-1, PR-10, plant defense responses 


\section{Introduction}

Hypericum perforatum L., popularly known as St. John 's wort, is a widely investigated medicinal plant with a history of more than 2000 years, which possesses anti-depressant, anti-inflammatory, antiviral, anti-cancer, and anti-bacterial pharmacological properties (Patočka J 2003; Tatsis et al. 2007; Saddiqe et al. 2010) . H. perforatum L. contains a wide range of biologically active secondary metabolites, including hypericin, hyperforin, xanthones, essential oils, phenolic acids and a broad array of flavonoids (Butterweck et al. 2003; Tatsis et al. 2007; Karppinen 2010; Saddiqe et al. 2010) . Hence, metabolic engineering could offer opportunities to maximize the production of valuable substances on an industrial scale . Although g enetic transformation of $H$. perforatum L. by Agrobacterium rhizogenes and biolistic bombardment has been reported, these protocols cannot achieve the requirements of functional genomic research (Hou et al. 2016).

A. tumefaciens is a gram-negative, soil-borne bacterium that causes crown gall disease on a broad range of host plants by integrating a transfer DNA (T-DNA) from a tumor-inducing plasmid into the host genome (Pitzschke 2013; Gohlke and Deeken 2014) . This feature has been widely exploited to introduce foreign genes into the plant for functional genomics and genetic engineering purposes. However, as a bacteri al pathogen, A. tumefaciens activates a number of defense responses in plants that include alteration $s$ in i) phytohormone metabolism (Pitzschke 2013), ii) pathogenesis-related protein synthesi s (Anand et al. 2007; Pruss et al. 2008) ， iii) microRNA-mediated gene silencing (Dunoyer et al. 2006), iv) lignin deposition (Miedes et al. 2014; Franklin et al. 2009) , v) secondary metabolite profile s (Franklin et al. 2009), and vi) production of reactive oxygen species (ROS) (Franklin et al. 2008).

A. tumefaciens-mediated transformation is the preferred method of gene transfer to plants, and it could be useful for metabolic engineering and functional genomic studies in $H$. perforatum $\mathrm{L}$. However, $H$. perforatum L. recalcitrance to A. tumefaciens-mediated transformation constitutes a major concern in the field (Hou et al. 2016). In a previous study, H. perforatum L. suspension cells showed an intense oxidative burst upon interaction with A.tumefaciens, with $99 \%$ mortality of A.tumefaciens taking place in few hours while no apoptotic process in plant cells was recorded (Franklin et al. 2008).

Phenolic oxidative coupling protein (Hyp-1) was first cloned and characterized from $H$. perforatum L. cell cultures (Bais et al. 2003). The cDNA sequence of Hyp-1 contains an open reading frame of 480 nucleotides, encoding a protein of 159 amino acids with a molecular weight of $17.8 \mathrm{kDa}$. Hyp-1 was considered as the sole enzyme that catalyzed the conversion of emodin to the key biocompound hypericin in H. perforatum L. in vitro (Bais et al. 2003), although other reports did not confirm this catalytic activity of th e protein (Košuth et al. 2007, 2011; Michalska et al. 2010; Karppinen et al. 2016). In addition, the highest expression level of Hyp-1 in H. perforatum L. was found in roots where no dark glands (sites of production of compounds of pharmacological value) are present, which left 
unresolved the function of Hyp-1 in the biosynthesis of hypericin (Košuth et al. 2007). Furthermore, it was observed that there was no clear correlation between hypericin production and Hyp- 1 expression, since the Hyp-1 gene was expressed in both "hypericin producing" and "hypericin lacking" species. These findings suggest that Hyp-1 is not a limiting factor in the biosynthesis of hypericin (Košuth et al. 2011).

In silico studies on the crystal structure of Hyp- 1 evidenced that Hyp- 1 belongs to the pathogenesisrelated (PR) class 10 family (Fernandes et al. 2008; Michalska et al. 2010; Sliwiak et al. 2016). PR-10 proteins are a family of small (154-163 amino acids, 15-18 KDa) and slightly acidic proteins which, unlike other PR proteins, accumulate in the cytosol . The major birch pollen allergen Bet v1 is considered the prototype for the PR-10 protein family and shares $45.1 \%$ homology to Hyp- 1 (Fernandes et al. 2013; Hsu et al. 2014; Xu et al. 2014; Sliwiak et al. 2016) . Some PR-10 proteins have been shown to have antifungal activities. For instance, MtPR-10 identified from Medicago truncatula had antifungal activity against Aphanomyces euteiches (Colditz et al. 2005). SsPR-10 from Solanum surattense inhibited hyphal growth of Pyricularia oryzae (Pungartnik et al. 2009) . ZmPR10.1 from Zea mays had antifungal activity against Aspergillus flavus (Xie et al. 2010). CsPR-10 from Crocus sativus inhibited the growth of Fusarium oxysporum (Gómez-Gómez et al. 2011) . Besides, some PR-10s also show ed antiviral and antibacterial activities. CaPR-10 from Capsicum annuum had antiviral activities against Tobacco mosaic virus (Park et al. 2004) . ZmPR-10 and ZmPR-10.1 conferred antibacterial activities against Pseudomonas syringae pv. tomato DC3000 (Xie et al. 2010). Moreover, the increased expression of Hyp-1 upon wounding and treatments with salicylic acid (SA) and abscisic acid (ABA) suggested its role in plant defense (Košuth et al. 2013; Karppinen et al. 2016). Furthermore, upon infection by A. tumefaciens, Hyp-1 expression was significant upregulated in all analyzed species of Hypericum (Košuth et al. 2013).

Gain-of-function approaches constitute useful tools for connecting genes to biological pathways.

Among them, overexpression of individual genes by genetic transformation has been used to identify genes that confer stress tolerance to plants (Kondou et al. 2010). There are also abundant reports on studies in plants overexpressing PR genes, resulting in enhanced tolerance to pathogen infection. In addition, 1 oss-of-function approaches have been exploited by plant pathologists as a parallel or complementary method to overexpression studies. Virus-induced gene silencing (VIGS) has been developed as a well-known technique based on RNA silencing to knock-down the expression of endogenous genes by utilizing recombinant viruses. Recently, Tobacco rattle virus (TRV)- based VIGS has been successfully used in the genetically non-tractable plant species Catharanthus roseus to investigate gene function (Liscombe and O'Connor 2011).

The aim of this study was to analyze the role of Hyp- 1 as a candidate involved in H. perforatum $\mathrm{L}$. recalcitrance to Agrobacterium tumefaciens-mediated transformation, and its impact on the expression of core genes regulating several defense pathways in Hyp- 1 transgenic tobacco plants. 


\section{Materials and methods}

\section{Plant materials and growth conditions}

Seeds of $N$. tabacum were surface-sterilized by immersion in $1.5 \%(\mathrm{v} / \mathrm{v})$ bleach for $7 \mathrm{~min}$, washed four times with sterile distilled water and sown onto $1 \%$ agar plates containing half strength Murashige and Skoog (MS) medium. The seeds of two varieties Batavia Laura (Casa César Santos, Portugal) and Salad bowl rossa (Vilmorin Jardin, France) of L. sativa were surface -sterilized by immersion in $6 \%(\mathrm{v} / \mathrm{v}) \mathrm{NaClO}$ for 15 and $10 \mathrm{~min}$, respectively, then washed four times with sterile distilled water and sown onto $1 \%$ agar containing half strength MS medium. All steps were done in sterile flow chamber and seeds were grown in the tissue culture room at $25^{\circ} \mathrm{C}$ with a $16-\mathrm{h} \mathrm{light} / 8-\mathrm{h}$ dark photoperiod.

\section{Suppression subtractive hybridization (SSH) and cDNA library construction}

Suspension cells of $H$. perforatum L. were treated with A.tumefaciens as described and a forward subtractive cDNA library was performed by using the PCR-Select cDNA Subtraction kit ( Clontech, USA) according to the manufacturer 's instructions. RNAs isolated from Hypericum suspension culture challenged with A. tumefaciens served as tester, while RNAs derived from control cultures served as driver, as described previously (Singh et al. 2016). Briefly, $2 \mu \mathrm{g}$ Poly A+ RNA from tester and driver were used to prepare cDNA pools. Both cDNA s were digested with RsaI to obtain shorter, blunt-ended molecules, followed by phenol/chloroform extraction, ethanol precipitation and finally resuspension in RNase-free water. Tester cDNAs were split into two equal pools, each of which was suitable for ligation with a different adapter, supplied in the kit. After first and second hybridizations, the purified subtracted cDNAs were cloned in to pGEM-T easy cloning vector (Promega, Madison, WI, USA). Individual colonies were screened by PCR for size of inserts, and fragments above $150 \mathrm{bp}$ were subjected to single-pass sequencing. Vector contamination was removed using the Vecscreen bioinformatics tool (http ://www.ncbi. nlm.nih.gov/tools/ vecscreen). The sequence similarity of obtained ESTs was analyzed by BLASTN and BLASTX and compared in different databases.

\section{Plasmid construction and site-directed mutagenesis}

For Hyp-1 overexpression, pBI121-GFP-Hyp-1 and pBI121-Hyp-1 were constructed. The full-length cDNA of the Green fluorescence protein (GFP) was amplified from the pGE vector (Received from Prof. Jim E. Brandle, Canada) (Conley et al. 2009) by Platinum Taq DNA Polymerase High Fidelity (Invitrogen, USA) with the following forward and reverse primers: BamHI-GFP-F and GFP-SpeI-SacIR. After sequencing, the GFP cDNA was digested with BamHI/SacI and inserted downstream of the Cauliflower mosaic virus (CaMV) 35S promoter in the binary vector pBI121 (GenBank Accession No.AF485783) to generate $\mathrm{pBI} 121-\mathrm{GFP}$. To produce Hyp-1 cDNA, a 480-nucleotide fragment was 
RT-PCR-amplified from the total RNAs of a $H$. perforatum L. suspension culture treated with $A$. tumefaciens, using Maxima First Strand cDNA Synthesis Kit (Thermo Scientific, USA) and Platinum Taq DNA Polymerase High Fidelity with primers SpeI-Hyp-1-F and Hyp-1-SacI-R. The sequenced Hyp-1 PCR fragment was finally cloned into the plasmid pBI121-GFP described above by digestion with SpeI/SacI to achieve construct pBI121-GFP-Hyp-1.

For the construction of pBI121-Hyp-1, the GFP gene was removed from pBI121-GFP-Hyp-1 by digestion with BamHI/SpeI. The Klenow Fragment of DNA Polymerase I (Invitrogen, USA) was used for producing blunt ends, and then the termini were ligated by T4 DNA Ligase (Thermo Scientific, USA).

To construct pBI121-GFP-Hyp-1-N, three stop codons (TAG TAA TGA) were added at 240 bp after the second ATG (nt 203) in the Hyp-1 sequence. To prepare the template pT-SpeI-Hyp-1-SacI, the plasmid pBI121-GFP-Hyp-1 and a self-ligated pGEM-T easy vector were digested by SpeI/SacI, and then ligated by T4 DNA ligase. To achieve pBI121-GFP-Hyp-1-N, the plasmid pT-SpeI-Hyp-1-SacI and the site-directed mutagenesis primers: STOP-Hyp-F and STOP-Hyp-R were used to amplify a pTStop codon-Hyp-1-N fragment using Platinum Taq DNA Polymerase High Fidelity. After the circularization, pT-Stop codon-Hyp-1-N was double digested with $\quad$ SpeI/SacI and inserted into pBII121-GFP digested with the same restriction enzymes downstream of GFP sequence.

All primer sequences are given in Table S1. All the purified recombinant plasmids were transformed into $A$. tumefaciens strain EHA105 by chemical transformation.

\section{Agrobacterium-mediated transformation}

Transgenic $N$. tabacum plants were generated by the leaf dis k transformation method as described by Horsch et al. (Horsch et al. 1985). Briefly, in vitro-grown N. tabacum leaves were cut into 4-6 pieces and laid on the MSIB medium (MS medium containing IAA $0.5 \mathrm{mg} / \mathrm{L} ; \mathrm{BA} 2.5 \mathrm{mg} / \mathrm{L}$ ) for 2 days under the dark in the tissue culture room . The explants were co-incubat ed with A.tumefaciens with an optical density at $600 \mathrm{~nm}\left(\mathrm{OD}_{600}\right)$ of 0.5-0.8 for $8 \mathrm{~min}$ under vacuum. Explants were kept in the same medium in the dark for another two days. Then explants were transferred into shooting medium (MSIB medium containing $50 \mathrm{mg} / \mathrm{L}$ Kanamycin and $500 \mathrm{mg} / \mathrm{L}$ ticarcillin), and re-cultured after every two-week period. Shoots $(3-4 \mathrm{~cm})$ were transferred into tubes with MS medium containing $0.5 \mathrm{mg} / \mathrm{L}$ IBA for root development.

For L. sativa transformation, two varieties Batavia Laura (BL) and Salad bowl rossa (SBR) were used in this study. Seedlings (4-6 days old) of L. sativa were punctured gently on the leaf surface near the petiolar region by scalpel blade, transferred to MS0.1BN (MS medium with $0.1 \quad \mathrm{mg} / \mathrm{L} \mathrm{BA}$ and 0.1 $\mathrm{mg} / \mathrm{L} \mathrm{NAA}$ ) and incubated in 16-h light/8-h dark condition at $25^{\circ} \mathrm{C}$ for 1 day. Explants were cocultivated with $A$. tumefaciens suspension at $\mathrm{OD}_{600}$ of around 0.5 under vacuum for $10 \mathrm{~min}$. Explants were then transferred to MS0.1BN plates under light for 5-7 days. Then explants were transferred to MS0.1BN medium with $50 \mathrm{mg} / \mathrm{L}$ Kanamycin $+500 \mathrm{mg} / \mathrm{L}$ ticarcillin, and re-cultured every two-weeks 
until initiation of shooting. Finally, explants were transferred to MS medium with 50

Kanamycin $+500 \mathrm{mg} / \mathrm{L}$ ticarcillin for rooting and waiting for harvesting the whole transgenic plants.

All the transgenic T1 plants were confirmed by PCR amplification of the Hyp-l and NPTII genes and by detection of expression of GFP fluorescence by microscopy (Leica - DM5000B + CTR5000 + ebq100, Germany). Progenies derived from self-fertilized T1 plants were germinated in MS medium containing $50 \mathrm{mg} / \mathrm{L}$ Kanamycin.

\section{Plant total RNA isolation and quantitative real-time RT-qPCR}

Total RNAs from young leaves of control and GFP-Hyp-1 transgenic N. tabacum plants were isolated by the CTAB method, as described (Gasic et al. 2004). The RNeasy Mini Kit (Qiagen, Germany) was used to clean up RNA including the DNase treatment according to the manufacturer 's instructions. Total RNAs were quantified by NanoDrop 1000 Spectrophotometer ( NanoDrop Technologies, Inc., USA) at A260/A280 and structural integrities were checked by $1.0 \%$ agarose-gel electrophoresis. $1 \mu \mathrm{g}$ of DNase treated RNA was used to perform cDNA synthesis by Maxima First Strand cDNA Synthesis Kit according to the manufacturer's instructions.

Gene expression was monitored by RT- qPCR in 96-well plates with 5x HOT FIREPol ${ }^{\circledR}$ EvaGreen ${ }^{\circledR}$ qPCR Mix Plus (ROX) (Solis Biodyne, Estonia). For each biological replicate, RT- qPCR reactions were performed in triplicate using $3 \mu 15 \mathrm{x}$ EvaGreen ${ }^{\circledR}$ qPCR master mix, $200 \mathrm{nM}$ of each primer, $1 \mu 1$ 10x diluted cDNA template and nuclease-free water to a final volume of $15 \mu 1$. Amplification was performed on CFX96 Real-Time System C1000 Thermal Cycler (Bio-Rad Laboratories, Inc., USA) with the following conditions: $95^{\circ} \mathrm{C}$ for $15 \mathrm{~min}, 45$ cycles of $95^{\circ} \mathrm{C}$ for $15 \mathrm{~s}, 60^{\circ} \mathrm{C}$ for $20 \mathrm{~s}$ and $72^{\circ} \mathrm{C}$ for $20 \mathrm{~s}$. Primers were designed to specifically anneal with each of the $N$. tabacum genes by using the online quantitative PCR primers design software QuantPrime (Arvidsson et al. 2008) . Gene expression was normalized with the internal control Elongation factor $1 \alpha(E F-1$ a) and L25 ribosomal protein genes (Schmidt and Delaney 2010). Primer efficiencies were determined using a 4-fold serial dilution of $1 \mu 1$ (10x diluted) cDNA template with 3 dilution points. The CFX Manager Software (BioRad Laboratories, Inc., USA) was used to analyze data. The relative quantification of PCR products was calculated by the $2^{-\lambda}{ }^{2} \mathrm{CT}$ method.

For microRNA detection, the method based on adding poly(A)-tailing to validate the expression of artificial microRNAs was employed (Shi et al. 2012) . For poly (A) tailing, $250 \mathrm{ng}$ of total RNA, $1.25 \mu 1$ 10mM ATP, $1 \mu 1$ Yeast Poly(A) Polymerase (1 U/ $\mu$ ) (Applied Biological Materials Inc, Canada), $5 \mu 1$ 5X Poly(A) Polymerase Buffer, $2.5 \mu 125 \mathrm{mM} \mathrm{MnCl} 2$ and Nuclease-free water up to 25 $\mu 1$ was mixed and the reaction mixture was incubated at $37^{\circ} \mathrm{C}$ for 20 minutes, followed by $65^{\circ} \mathrm{C}$ for 20 minutes to terminate the reaction. cDNA was synthesized as follows: $25 \mu 1$ Poly (A) RNA was added to 25 pmol of polyT adapter (Shi et al. 2010) and the mixture was incubated at $70^{\circ} \mathrm{C}$ for $2 \mathrm{~min}$ followed by cooling on ice for $2 \mathrm{~min}$. Reactions were then mixed with $8 \mu 1$ of $5 \mathrm{X}$ First-Strand Buffer, 
$1 \mu 1$ of dNTP Mix (10 mM each), $1 \mu 1$ of DTT (20 mM), $0.5 \mu$ l SMARTScribe Reverse Transcriptase (Clontech, USA) and sterile water up to $40 \mu \mathrm{l}$. The reaction mixture was incubated at $42^{\circ} \mathrm{C}$ for $1.5 \mathrm{hr}$. Diluted cDNA template ( $1 \mu 15 \mathrm{x}$ diluted) was used to conduct the qPCR reactions with gene-specific primers as described above. Gene expression was normalized to the $N$. tabacum 5.8S rRNA gene using (Shi et al. 2010). Data was analyzed as described above.

To detect the relative accumulation of $C r P R-1$ and $C r P R-5$ genes, one-step RT-qPCR was carried out in the Rotor-Gene 6000 real-time PCR detection system (Corbett, Australia) using CrPR-1- and CrPR5 -specific primers. To ensure the absence of DNA template in the samples, RT-qPCRs with and without reverse transcriptase were run in parallel, and

C. roseus $40 \mathrm{~S}$ ribosomal protein $\$ 9$ gene (CrRSP9) (Sheshadri et al. 2018) was used to normalize gene expression. RT-qPCR was carried out at $50^{\circ} \mathrm{C}$ for $10 \mathrm{~min}, 95^{\circ} \mathrm{C}$ for $3 \mathrm{~min}, 40$ cycles of $95^{\circ} \mathrm{C}$ for $10 \mathrm{~s}$, and $60^{\circ} \mathrm{C}$ for $20 \mathrm{~s}$. The efficiency of each pair of primers was determined by using a 5-fold serial dilution starting from $100 \mathrm{ng} / \mu 1 \mathrm{RNA}$, with five dilution points. The Rotor-Gene 6000 software was used to confirm the synthesis of cDNA products by melting curves. All primer sequences are given in Table S1.

\section{GUS staining assays}

Fresh leaf disks were collected by sterile core borer or cut into pieces of rhombus-like shape. The disks were sterilized with $70 \%$ ethanol for $30 \mathrm{~s}, 6 \% \quad \mathrm{NaClO}$ for $90 \mathrm{~s}$, followed by washing with sterilized water. Leaf disks were kept on MSIB medium in the plant culture room for 2 days. The leaf disk transformation method was used to introduce Agrobacterium strain EHA105 harboring pCAMBIA2301-GUS into plant tissues. After 5-6 days, leaf disks were incubated in the GUS staining solution ( $0.5 \mathrm{mg} / \mu 1 \mathrm{X}$-Gluc (5-bromo-4-chloro-3-indolyl ־D-glucuronide cyclohexylammonium salt), 20 mM sodium phosphate buffer (pH 7.0), 10 mM EDTA (pH 8.0), 5 mM K4FeCN6 and 5mM $\mathrm{K} 3 \mathrm{FeCN} 6$ ) at $37^{\circ} \mathrm{C}$ overnight. Next day, the samples were washed by $70 \%$ ethanol and the GUS activity was viewed and photographed.

\section{Subcellular localization}

For Hyp-1 subcellular localization assays, A. tumefaciens cultures harboring pBI121-GFP-Hyp-1 and the mCherry-labeled-plasma membrane marker PM- RK (Nelson et al. 2007) were co-infiltrated into the leaves of 5-week-old N. tabacum plants as described previously (Hou et al. 2011) . Confocal fluorescence of GFP and mCherry were captured with Leica TCS SP2 confocal microscope. Excitation and emission settings in nanometers $(\mathrm{nm})$ were 488 and 500 -to-530 respectively for GFP; 561 and 575-to-640 respectively for mCherry.

To distinguish the localizati on of Hyp-1 between cell wall and plasma membrane, $N$. tabacum leaves were agroinfiltrated with pBI121-GFP-Hyp-1 and free GF P as contr ol. After 3 days, 1- $\mathrm{cm}^{2}$ leaf explants were excised and incubated with $1 \mathrm{M}$ mannitol for $5 \mathrm{~min}$ prior to observation. GFP 
fluorescence patterns were examined in epidermal cells with Leica DM5000B+CTR5000+ebq100 fluorescence micros cope. Images were processed with ImageJ (Schindelin et al. 2012) and Adobe Photoshop software (Adobe Systems).

\section{Virus-induced gene silencing}

For VIGS, TRV-based vectors , pTRV1 and pTRV2:00, were obtained from ABRC (http://www.arabidopsis.org). pTRV2: CrChlhI containing a fragment of C. roseus protoporphyrin IX magnesium chelatase subunit $H(\mathrm{ChlH})$ gene served as marker to evaluate VIGS efficiency.

A series of amino acid sequences from Hyp- 1 related genes were aligned to generate a phylogenetic tree by MEGA 6 (Tamura et al. 2013). The homologous Hyp-1 gene from C. roseus intracellular pathogenesis related protein (CrIPR) (GenBank: Y10612.1) was selected to construct pTRV2: CrIPR. A 318-bp fragment from the 3' terminus of CrIPR was used to synthesize cDNA by Superscript II reverse transcriptase (Invitrogen, USA). The cDNA was amplified with $\quad$ Cr-IPR-specific primers by High-fidelity Taq DNA Polymerase (Roche, Germany). The PCR product was first cloned in pGEM-T easy vector. After DNA sequencing, the EcoRI-digested CrIPR fragment was inserted into pTRV2:00, which was previously digested with EcoRI followed by dephosphorization with alkaline phosphatase (Roche, Germany) according to the manufacturer 's instructions. All the recombinant plasmids for VIGS assays were transformed into $A$. tumefaciens strain GV3101 by electroporation.

Three-to-four week-old C. roseus plants (Vilmorin Jardin, France) having two pairs of fully developed true leaves were used for agroinfiltration of viral vectors as described previously (Liscombe and O’Connor 2011).

Silencing of CrIPR mRNA was detected in upper, non-inoculated leaf tissue at 21 days postinoculation (dpi) by semiquantitative RT-PCR, as described (Liscombe and O 'Connor 2011). Cr-IPR(C)-2-specific primers that anneal outside the region targeted for silencing were used to ensure that the endogenous gene would be tested. The CrRSP9 described above was used as internal control. PCR cycles included denaturation at $98^{\circ} \mathrm{C}$ for $30 \mathrm{~s}$, annealing at $58^{\circ} \mathrm{C}$ for $20 \mathrm{~s}$, and elongation at $72{ }^{\circ} \mathrm{C}$ for 30 s. Aliquots were removed from the thermocycler after 36, 39, and 42 cycles. All primer sequences are given in Table S1.

\section{Western blotting}

A. tumefaciens carrying pCAMBIA2300 containing a free GFP reporter gene was used to challenge silenced and control C. roseus plants. Samples were collected at 3 dpi and 5 dpi and western blot was carried out to measure GFP expression. Total proteins were extracted from $100 \mathrm{mg}$ plant leaves in 400 $\mu 1$ extraction buffer containing $0.1 \mathrm{M}$ Tris- $\mathrm{HCl}$ ( $\mathrm{pH} 8.0$ ), 10mM EDTA ( $\mathrm{pH}$ 8.0), $0.1 \mathrm{M} \mathrm{LiCl}, 1 \%$ SDS and $1 \%$ fresh added $\square$-mercaptoethanol. Samples were separated on $15 \%$ SDS-PAGE gels and transferred to poly- (vinylidene difluoride) ( PVDF) membranes (Amersham, GE Healthcare, Little Chalfont, Buckinghamshire, UK). The membranes were blotted with rabbit anti-GFP, N-terminal 
antibody (Sigma-Aldrich, St Louis, MO, USA). Blotted proteins were detected by secondary antibody Goat anti-Rabbit IgG HRP (Sigma-Aldrich, USA) and LiteAblot ${ }^{\circledR}$ PLUS Enhanced Chemiluminescent Substrate (Euroclone, Italy).

\section{Statistical analysis}

The expression levels of target genes involved in different plant defense pathways were compared by One-way ANOVA. The relative accumulation of $P R$ genes in $C$. roseus was analyzed by Student's ttest using GraphPad Prism 6 (GraphPad Software, Inc., USA).

\section{Results}

\section{Overexpression of Hyp-1 in N. tabacum and L. sativa}

To identify genes from $H$. perforatum L. involved in defense responses against $A$. tumefaciens, a SSHbased cDNA librar y was performed (Singh et al. 2016) . RNAs isolated from H. perforatum L. suspension cells challenged with A. tumefaciens as tester and with RNAs from control cultures as driver were used to construct the forward subtractive cDNA library from which a highly expressed Hyp-1 gene was selected (Table S2) (Bais et al. 2003).

To characterize Hyp-1 function in defense response s, we employed the Agrobacterium susceptible model plant $N$. tabacum to establish transgenic plants expressing Hyp-1 fused at the N-terminus to GFP under the control of 35S promot er in the binary expression vector pBI121 (Fig . S1). Four transgenic tobacco plant lines, GFP-Hyp-1-2/-3/-4/-5, were selected by antibiotic resistance , and TDNA insertion was confirmed by PCR. As control, besides wild type (WT), transgenic tobacco plants expressing GFP were obtained.

In order to determine whether overexpression of Hyp-1 influences the efficiency of Agrobacteriummediated expression in tobacco, we inoculated two T2 progenies derived from each of three $\mathrm{T} 1$ lines, GFP-Hyp-1-3/-4/-5, with the strain EHA105 harboring a binary plasmid to express GUS . Whereas GFP transgenic control plants showed an intense GUS staining and behaved similarly to WT plants (Fig. 1 b, c), GUS staining was absent in either of the six GFP-Hyp-1 T2 transgenic lines (Fig. 1 d-i) or WT plants inoculated with A. tumefaciens containing the empty vector (Fig. 1 a).

Next, we assessed Agrobacterium-mediated expression in two varieties of other Agrobacterium susceptible plant L. sativa, BL and SBR, overexpressing GFP-Hyp- 1 and Hyp- 1 respectively (Fig S2 and S3), by inoculation with Agrobacterium harboring the GUS gene. T1 transgenic plants expressing either GFP-Hyp-1 or Hyp-1 showed no staining at all (Fig. 2 d, f, and Fig. 3 c) or a clear decrease in GUS staining (Fig. 2 c, e) compared to WT plants (Fig. 2 b and 3 b). Our results indicate that expression efficiencies by $A$. tumefaciens greatly decrease in tobacco and lettuce plants constitutively overexpressing Hyp-1, suggesting the involvement of Hyp-1 in defense response against A. tumefaciens. 
The conserved motif Bet.v.1 positioned at the C -terminal part of Hyp-1 (89-120 amino acid residues in Hyp-1) is common throughout the PR-10 proteins, suggesting a prominent role in protein function (Bais et al. 2003) . However, there are no studies on Hyp-1 protein integrity nor function of the Nterminus of Hyp-1 up to now. So, to study the contribution of the N-terminal part of Hyp-1 and the protein integrity of Hyp-1 to the defense response against A. tumefaciens, we generated transgenic tobacco plants expressing the N-terminal 80 amino acids of Hyp-1 fused to GFP (Fig. S4) (GFP-Hyp1-N plants). Three T2 progenies derived from each of two T1 lines, GFP-Hyp-1-N-2/- 3, were inoculated with the A. tumefaciens strain harboring the GUS gene. Interestingly, T2 transgenic plants expressing GFP-Hyp-1-N showed an intense GUS staining comparable to that in WT control plants (Fig. $4 \mathrm{~b}$ and $4 \mathrm{Cch}$ ). This suggests that the $\mathrm{N}$-terminal region of Hyp-1 is not sufficient to function as a defense protein against $A$. tumefaciens.

\section{Subcellular localization studies}

To examine the subcellular localization of Hyp-1， Agrobacterium strains carrying either GFP-Hyp-1 or the plasma membrane marker PM-RK were co-expressed transiently in leaf epidermal cells of WT $N$. tabacum by agroinfiltration. Two days after agroinfiltration, the fluorescence of both proteins was observed by confocal fluorescence microscopy to compare their localization. The fluorescence of GFPHyp-1 coincided with that of PM-RK in the plasma membrane (Fig. 5). Besides, GFP-Hyp-1 was also detected in the nucleus, but not in nucleolus, and in the cytoplasm of leaf epidermal cells of $N$. tabacum. The antibacterial defense activity of the Hyp-1 protein is retained in the GFP-Hyp-1 fusion protein (Figs. 1-3), suggesting that this subcellular localization of GFP-Hyp-1 has biological relevance.

In order to test whether Hyp-1 localized also to the cell wall, we induced cell plasmolysis by treatment of the leaf sections with $1 \mathrm{M}$ mannitol to promote the detachment of the plasma membrane from the cell wall (Fig. 6 d-f, j-o), using treatment with $\mathrm{H}_{2} \mathrm{O}$ as control (Fig. 6 a-c, g-i). In plasmolyzed cells GFP-Hyp-1 remained in the area lining the detached plasma membrane, suggesting that it was probably not present in the cell wall (Fig. 6 j-o).

We next investigated the subcellular localization of GFP-Hyp-1 in the GFP-Hyp-1-4/-5 transgenic tobacco lines. Green fluorescence was localized in the cytoplasm and nucleus of GFP-Hyp-1 expressing plants (Fig. 7 b-d), similar to the localization of transiently-expressed free GFP (Fig. 7 a). The integrity of the GFP-Hyp-1 fusion in the GFP-Hyp-1 transgenic plants was confirmed by western blot using antibodies against GFP (Fig. 7 e).

\section{Overexpression of Hyp-1 alters expression of multiple defense-related genes}

To survey plant defense pathways involve $\mathrm{d}$ in Hyp-1-mediated defense against A.tumefaciens, the expression levels of core genes implicated in different microbial defense pathways were determined in 
GFP-Hyp-1 transgenic tobacco plants by RT-qPCR. T2 GFP-Hyp-1-3, GFP-Hyp-1-4 and GFP-Hyp-15 transgenic lines were selected for subsequent experiments since they exhibited higher levels of Hyp1 mRNA than GFP-Hyp-1-2 (Fig. 8 a).

Phytohormones such as auxin and jasmonic acid (JA) play an important role in plant defense against pathogenic microorganisms. We focused on the auxin-responsive genes transport inhibitor response I(NtaTIR I) and auxin responsive factor 8 (NtaAFR8), and the JA biosynthesis gene Lipoxygenase1 (NtaLOX). The transcript levels of NtaTIRI and NtaARF8 were significantly reduced in Hyp-1 overexpression plants wh ereas NtaLOX did not show significant differences in two out of three transgenic lines tested, compared to WT control plants (Fig. 8 b-d).

PR proteins have been described as plant specific proteins that accumulate in response to pathogen interaction. To assess the involvement of PR proteins in Hyp-1-mediated antibacterial defense, the transcript levels of NtaPR-10 and NtaPR-1 were monitored by RT-qPCR (Fig. 8 e-f). Both NtaPR-1 and NtaPR-10 mRNAs were decreased in most of the GFP-Hyp-1 lines, especially NtaPR-1.

The microRNA-mediated gene silencing pathway is also involved in plant defense responses against pathogens. The key component in the miRNA pathway ARGONAUTE 1 (NtaAGO1), as well as NtamiR160, NtamiR164ab and NtamiR164c were analyzed by RT-qPCR in Hyp-1 overexpressing plants (Fig. 8 g-j). Results indicate that NtaAGO1 and Nta164ab were significantly reduced in all transgenic lines analyzed, whereas NtamiR160 was reduced in GFP-Hyp-1-4 and GFP-Hyp-1-5 lines which displayed the higher expression level of Hyp-1 (Fig. 8 a). There were no significant differences in NtamiR164c expression between transgenic and control plants.

Production of secondary metabolites via the phenylpropanoid pathway serves in plants as a defense mechanism to protect themselves from pathogens. The 1 ignin biosynthesis genes Phenylalanine ammonia-lyase 1, 4 (NtaPAL1, NtaPAL4), Cinnamoyl CoA reductase (NtaCCR) and Cinnamyl alcohol dehydrogenase (NtaCAD) were analyzed in Hyp-1 overexpressing plants by RT-qPCR. Transcripts of NtaPAL1,4 genes were downregulated in the Hyp-1 transgenic lines, while the expression levels of $N t a C C R$ and $N t a C A D$ were not significantly different to those in control plants (Fig. 8 k-n). The transcripts of Chalcone synthase (NtaCHS), a key enzyme which catalyzes the first committed step in the flavonoid biosynthesis pathway, was drastically reduced in Hyp- 1 overexpressing plants (Fig. 8 o).

Lastly, $\mathrm{t}$ ranscripts of core enzymes involved in ROS homeostasis, i.e., ascorbate peroxidase (NtaAPX), catalase (NtaCAT) and superoxide dismutase (NtaFe-SOD), were also monitored in Hyp-1 overexpressing plants by RT-qPCR. Results showed no significant differences in NtaAPX and NtaCAT expression between transgenic and control plants (Fig. 8 p, q), whereas the levels of NtaFe-SOD mRNA were downregulated in two out of three transgenic plants compared to control plants (Fig. 8 r).

\section{Virus-induced gene silencing in $C$. roseus}

To investigate the loss-of function phenotype of Hyp-1, we used the VIGS technique to knock-down 
the expression of the Hyp- 1 homolog in C. roseus. We choose C. roseus because is a genetically nontractable plant species that resembles the recalcitrance of $H$. perforatum L. to Agrobacterium infection (Di Fiore et al. 2004) . The Hyp-1 homolog in C. roseus is intracellular pathogenesis related protein $(C r I P R)$, as revealed by phylogenetic analysis of Hyp-1-related genes (Fig. S5). 3-4 weeks-old C. roseus plants were agroinfiltrated with a TRV-based VIGS vector carrying a 318-bp fragment of CrIPR (pTRV2: CrIPR) or the empty TRV vector (pTRV2:00) as a control. Silencing of the magnesium chelatase subunit $H(C h l H)$ gene, which provides a visual bleached leaf phenotype, was also used to monitor VIGS in C. roseus. At 21 dpi, photobleaching occurred in the third and fourth pairs of leaves in all pTRV2: CrChlH treated plants, whereas there was no visible phenotype in control and pTRV2: CrIPR infected plants (Fig. 9 a). Silencing efficiency of CrIPR was examined by semiquantitative RT-PCR in upper leaves equivalent to those showing the bleached phenotype in pTRV2: CrChlH infected plants . Transcripts of CrIPR was reduced substantially in pTRV2: CrIPR infected plants compared to pTRV2:00 infected, control plants (Fig. 9 b).

In order to determine whether the knock-down of CrIPR influences the efficiency of Agrobacteriummediated expression in C. roseus, CrIPR-silenced and control plants were inoculated in the third pairs of leaves with an A.tumefaciens strain expressing GFP at 21 dpi. GFP expression was monitored in duplicated samples at 3 and 5 dpi by western blot (Fig. 9 c). Intriguingly, GFP accumulated at lower levels in CrIPR-silenced plants than in controls at both time points. Our result indicates that expression efficiency by $A$. tumefaciens is decreased in CrIPR-knocked-down plants.

It has been reported that silencing of PR-10-like proteins in M. truncatula led to induction of other PR proteins and increased tolerance to the oomycete A. euteiches (Colditz et al. 2007). Thus, the relative accumulation of another set of PR protein genes from C. roseus, $C r P R-1$ and $C r P R-5$, were monitored in $C r I P R$-silenced and control plants by RT-qPCR. Interestingly, no significant difference in the expression of $C r P R-1$ mRNA was found between $C r I P R$-knock-down and control plants, whereas a $40 \%$ increase of $C r P R-5$ expression was measured in $C r I P R$-silenced plants compared with control plants (Fig. 9 d). These findings suggest a crosstalk between different defense responses in $C r I P R$ silenced C. roseus plants, which led to the up-regulation of $C r P R-5$.

\section{Discussion}

Hyp- 1 was selected from a forward subtractive cDNA library of $H$. perforatum L. suspension cells treated with A.tumefaciens. The function of Hyp-1 as a member of the PR-10 gene family has been barely studied (Košuth et al. 2013). Hyp-1 overexpression was carried out in tobacco as well as in two varieties of lettuce, two plant species amenable for A.tumefaciens-mediated transformation, to unveil its participation in defense against A. tumefaciens. Expression efficiencies of A. tumefaciens greatly decreased in all transgenic plants as assayed by histochemical GUS staining, indicating that Hyp- 1 participates in defense responses against A.tumefaciens. Previous findings indicated that GUS 
staining was a good indicator of Agrobacterium viability, suggesting that bacteria survival was compromised in Hyp-1 overexpressing plants (Franklin et al. 2008 ). In addition, $t$ ransgenic tobacco plants expressing a C-terminal truncated version of the protein were used to study the contribution of the first 80 amino acids to the antibacterial activity of Hyp- 1. Defense activit ies towards $A$. tumefaciens were highly reduced in GFP-Hyp-1-N transgenic plants, suggesting that the $\mathrm{N}$ terminal part of Hyp-1 is not enough to contribute to the biological function of the Hyp-1 protein. The importance of maintaining Hyp-1 protein integrity could be interpreted in the light of studies on the crystal structure of Hyp-1 (Michalska et al. 2010). The Hyp-1 molecule exhibits a typical PR-10-like protein structure, whose fold consists of seven-stranded antiparallel $\square$-sheet wrapped around the Cterminal helix $\square$. Together with another two $\square$-helixes, those main structural elements form a huge cavity within the protein interior to bound various hydrophobic ligands, like plant hormone cytokinin . Besides, a characteristic Bet.v.1 domain is positioned at 89-120 amino acid residues in Hyp-1 (Bais et al. 2003). Therefore, several of those structural motifs are lacking in the Hyp-1 mutant, which results in the loss of biological activity.

Hyp-1 protein was localized at the plasma membrane, nucleus and cytoplasm, as judged by the subcellular distribution of fluorescence derived from the transient expression of GFP-Hyp-1 in $N$. tabacum leaves. Moreover, confirmation of the integrity of the full-length fusion protein in GFP-Hyp1 transgenic plants by Western blot confirmed that the nuclear visualization of GFP fluorescence was due to Hyp-1 accumulation in nucleus rather than the import of free GFP cleaved from the fusion protein. This study demonstrates for the first time the subcellular localization of Hyp- 1 in nucleus , as had been reported for the PR-10 protein s from Tamarix hispida and Vitis vinifera (Zhang et al. 2010; He et al. 2013). The nuclear localization of Hyp-1 might play an important role in defense responses against invading pathogens, especially in pathogens like A. tumefaciens, whose virulence depends on integration of T-DNA into the nucleus.

To further corroborate the role of Hyp- 1 in defense responses against A. tumefaciens, we used a TRVbased VIGS approach to study the loss-of- function phenotype of the Hyp- 1 homologous CrIPR in the recalcitrant plant $C$. roseus. Contrary to what could be expected, Agrobacterium-mediated expression efficiency was decreased in $C r I P R$-silenced plants, which correlated with the up-regulation of $C r P R-5$. It was reported that M. truncatula transgenic lines silenced for $P R-10$ showed a reduced colonization of the oomycete A. euteiches, and a suppressed infection in the roots of transgenic plants (Colditz et al. 2007). Interestingly, $M t P r-10$ silencing led to the de novo induction of two isoforms of thaumatinlike proteins (PR-5b). Thus, antagonistic induction of PR -5 proteins, which are normally repressed due to PR-10 expression, is not a n unprecedented phenomenon in plant-microbe interactions. A similar antagonistic crosstalk was observed in our Hyp- 1 overexpressing tobacco lines, where overexpression of $P R-10$ from H. perforatum L. led to reduced expression of endogenous PR genes, $P R-1$ and PR-10. In addition, silencing of Polygalacturonase-inhibiting protein in N. benthamiana 
greatly decreased Agrobacterium-mediated expression efficiency, which correlated with induction of several PRs, including PR-5 (Hou et al. submitted). These findings illustrate the complex crosstalk regulating multi-factorial defense responses in plant-pathogen interactions.

A. tumefaciens as a microbial pathogen provoke $\mathrm{s}$ a series of plant defense responses, but understanding how these responses affect pathogen infection is still unclear (Pitzschke 2013). To survey which plant defense responses could be involved in Hyp-1-mediated defense against $A$. tumefaciens, the expression levels of core genes from different pathways including auxin metabolism, microRNA-mediated gene silencing, lignin deposition, secondary metabolites, ROS signaling, and pathogenesis-related proteins, were determined in GFP-Hyp-1 tobacco plants. Auxin as a phytohormone is a critical contributor in plant-p athogen interaction (Fu and Wang 2011). There are two groups of protein complexes in auxin signaling: $t$ he auxin signaling repressor auxin/indole acetic acid (Aux/IAA) and the auxin signaling receptor transport inhibitor response 1/auxin signaling F-box proteins (TIR1/AFB). Pathogen-produced IAA is perceived by TIR1 and activates TIR1/AFB to degrade Aux/IAA which negatively regulates auxin signaling by binding and inhibiting auxin responsive factors (ARFs). The released ARFs then regulate transcription of auxin-responsive genes. In addition, plant IAA biosynthesis can be up-regulated by some ARFs, and the increased IAA level causes cell wall expansion by inducing the expression of structural proteins like endo- $\square-1,4-$ glucanases (EGases) and xyloglucan endotransglycosylases (XETs). These activities participate in cell wall loosening that promotes pathogen invasion and disease development . Moreover, auxin is involved in stomata opening that allows some pathogens to gain access to the interior of plant tissues. However, plants evoke mechanisms to repress auxin signaling during pathogen infection as a defense strategy (Fu and Wang 2011). In the present study, transcripts of auxin-responsive genes NtaTIRI and NtaAFR8 were remarkably reduced when Hyp-1 was overexpressed, which suggests that Hyp-1 overexpression could contribute to antibacterial defenses by reducing auxin signaling. Besides, overexpression of Hyp- 1 in transgenic plants led to a decrease in the transcriptional levels of NtaAGO1 and two microRNAs , NtamiR160 and NtamiR164ab. In A. thaliana, miR164 has been reported to target the transcripts of NAM/ATAF/CUC (NAC) domain protein $N A C 1$, which acts downstream of TIRI to transmit auxin signals for lateral root emergence (Guo et al. 2005). A recent report suggested that miR164 and target gene NAC4 regulate cell death during plant-pathogen interactions, possibly by repres sing GDSL lipase 1 which is an important regulator of plant immune responses (Lee et al. 2017). MiR164 is also involved in plant response to abiotic stresses in Brassica, rice and potato (Fang et al. 2014; Bhardwaj et al. 201 5; Zhang et al. 2018) . MiR160 target s ARF10, $A R F 16$ and $A R F 17$ to down regulate auxin signaling, which in turn protect plants against bacteria and fungi (Pinweha et al. 2015; W ójcik et al. 2017) . Our results offer preliminary evidence on the involvement of those two microRNAs in the regulation of plant defense against A. tumefaciens that needs to be further investigated. 
Several key genes involved in the lignin and flavonoids biosynthesis pathways, NtaPAL1,4, NtaCCR, NtaCAD and NtaCHS, were monitored in Hyp-l overexpressing plants . PAL is the first enzyme of phenylpropanoid pathway, which comprises the synthesis of lignin and flavonoids

(Heldt and Piechulla 2011; Bagal et al. 2012) . Lignin, as a critical constituent of cell wall, plays a major role in structural defense against pathogens. Flavonoids are small molecular secondary metabolites acting as antimicrobial compounds in defense against pathogens (Mazid et al. 2011; Andersson 2012; Goyal et al. 2012). Our findings showed that Hyp-l overexpression in transgenic plants downregulated the expression of genes involved in the phenylpropanoid pathway ( NtaPAL1,4 and NtaCHS), whereas NtaCCR and NtaCAD were not changed, suggesting that overexpressing of Hyp-1 decreased Agrobacterium-mediated expression efficiency independently from synthesis of lignin and flavonoids. Similarly, ROS signaling did not seem to play a significant role in Hyp-1-mediated defense against $A$. tumefaciens since the transcript levels of core enzymes were unaltered (NtaAPX, NtaCAT) or slightly repressed (NtaFe-SOD) in Hyp- 1 overexpressing plants. Nevertheless, although Hyp- 1 overexpression could induce wide changes in the gene expression pattern of defense genes, not all of these changes are necessarily involved in the molecular mechanisms underlying Hyp-1-mediated antibacterial defense.

Several PR-10 proteins have shown in vitro and in vivo to have DNase and RNase activities, which could help to explain the role of Hyp-1 in antibacterial defense (Chen et al. 2006; Kim et al. 2008, 2011; Xie et al. 2010; Krishnaswamy et al. 2011; He et al. 2013) . Chadha and Das (Chadha and Das 2006) reported that an RNase activity-competent version of PR-10 from peanut was internalized by hyphae of Fusarium oxysporum and inhibited fungal growth. Moreover, DNA fragmentation was detected in rice suspension-cultured cel ls treated with PBZ1, a rice PR -10 family protein (Kim et al. 2011). Therefore, the putative DNase and RNase activities of Hyp-1 might also play an important role in the death of A. tumefaciens cells observed upon co-cultivation with $H$. perforatum L. (Franklin et al. 2008). In addition, our subcellular localization data suggest that Hyp-1 localized to the plasma membrane. The plasma membrane is important during plant-microbe interactions, because it acts as a barrier between the host and the pathogen and many pathogen-associated molecular pattern recognition receptors are localized at the plasma membrane (Boller and Felix 2009) . Other PR proteins with associated RNase activities have been localized in the cell plasma membrane (Jiang et al. 2015).

In summary, Hyp-1 was selected from a forward subtractive cDNA library from

A. tumefacienstreated $H$. perforatum L. suspension cells. Its biological function in defense against A. tumefaciens was analyzed in transgenic Nicotiana tabacum and Lactuca sativa plants overexpressing Hyp-1, and in C. roseus silenced for the Hyp-1 homologous gene. Moreover, the expression of core genes regulating several defense pathways was analyzed in Hyp- 1 overexpressing lines. Hyp- 1 was detected in the nucleus, plasma membrane and the cytoplasm of epidermal cells by confocal microscopy. Overall, our 
findings suggest that Hyp-1 from $H$. perforatum L. modulates its recalcitrance to A.tumefaciensmediated transformation as part of a multi-factorial response against pathogen infection. Future research on the characterization of RNase and DNase activities of Hyp-1 might shed light on its protective role in degrading DNA or RNA of invading pathogens in H. perforatum L. Agrobacteriummediated genetic transformation approaches that reduce Hyp- 1 expression in $H$. perforatum L. could give a better chance at efforts aimed at the genetic manipulation of this recalcitrant and medicinal plant spp. However, antagonistic regulation of PR proteins and that of other inducible defense-related proteins that result in decreased Agrobacterium-mediated expression efficiency should be taken into consideration. In conclusion, $t$ he present study provides a better understanding regarding the functional characterization of Hyp-1 gene from $H$. perforatum L., and adds significant knowledge about the multi-factorial nature of the $H$. perforatum L. $-A$. tumefaciens interaction.

\section{Acknowledgements}

The author acknowledges the financial support provided by the FCT-Portuguese Founda tion for Science and Technology, grant UID/BIA/04050/2013 (POCI-01-0145FEDER-007569), PhD grant (SFRH/BD/52561/2014) under the Doctoral Programme "Agricultural Production Chains - from fork to farm" (PD/00122/2012) , and the Ministry of Economy and Competitiveness of Spain, grant BIO2016-75619-R (AEI/FEDER, UE) to T.C. and F.T.

We gratefully acknowledge the advice of Prof. Vincent L. Chiang and Dr. Rui Shi (North Carolina State University) for microRNA detection. We gratefully thank Prof. Mariana Sottomayor and Dr Joana Guedes (University of Porto) for kindly providing pTRV2: CrChlh and advice.

\section{References}

Anand A, Uppalapati SR, Ryu C-M, et al (2007) Salicylic acid and systemic acquired resistance play a role in attenuating crown gall disease caused by Agrobacterium tumefaciens. Plant Physiol 146:703-715. doi: 10.1104/pp.107.111302

Andersson P (2012) Secondary metabolites associated with plant disease, plant defense and biocontrol. Dissertation, Swedish University of Agricultural Sciences

Arvidsson S, Kwasniewski M, Riaño-Pachón DM, Mueller-Roeber B (2008) QuantPrime - A flexible tool for reliable high-throughput primer design for quantitative PCR. BMC Bioinformatics 9:1-15. doi: 10.1186/1471-2105-9-465

Bagal UR, Leebens-Mack JH, Lorenz WW, Dean JFD (2012) The phenylalanine ammonia lyase (PAL) gene family shows a gymnosperm-specific lineage. BMC Genomics 13:S1. doi: 10.1186/1471-2164-13-S3-S1

Bais HP, Vepachedu R, Lawrence CB, et al (2003) Molecular and biochemical characterization of an 
enzyme responsible for the formation of hypericin in St. John's wort (Hypericum perforatum L.). J Biol Chem 278:32413-32422. doi: 10.1074/jbc.M301681200

Bhardwaj AR, Joshi G, Pandey R, et al (2015) A genome-wide perspective of miRNAome in response to high temperature, salinity and drought stresses in Brassica juncea (Czern) L. PLoS One 10(4): e0125644. doi: 10.1371/journal.pone.0125644

Boller T, Felix G (2009) A renaissance of elicitors: perception of microbe-associated molecular patterns and danger signals by pattern-recognition receptors. Annu Rev Plant Biol 60:379-406. doi: 10.1146/annurev.arplant.57.032905.105346

Butterweck V, Christoffel V, Nahrstedt A, et al (2003) Step by step removal of hyperforin and hypericin: activity profile of different Hypericum preparations in behavioral models. Life Sci 73:627-639. doi: 10.1016/S0024-3205(03)00314-X

Chadha P, Das RH (2006) A pathogenesis related protein, AhPR10 from peanut: an insight of its mode of antifungal activity. Planta 225:213-222. doi: 10.1007/s00425-006-0344-7

Chen ZY, Brown RL, Rajasekaran K, et al (2006) Identification of a maize kernel pathogenesisrelated protein and evidence for its involvement in resistance to Aspergillus flavus infection and aflatoxin production. Phytopathology 96:87-95. doi: 10.1094/PHYTO-96-0087

Colditz F, Braun HP, Jacquet C, et al (2005) Proteomic profiling unravels insights into the molecular background underlying increased Aphanomyces euteiches -tolerance of Medicago truncatula . Plant Mol Biol 59:387-406. doi: 10.1007/s11103-005-0184-Z

Colditz F, Niehaus K, Krajinski F (2007) Silencing of PR-10-like proteins in Medicago truncatula results in an antagonistic induction of other PR proteins and in an increased tolerance upon infection with the oomycete Aphanomyces euteiches . Planta 226:57-71. doi: 10.1007/s00425006-0466-y

Conley AJ, Joensuu JJ, Jevnikar AM, et al (2009) Optimization of elastin-like polypeptide fusions for expression and purification of recombinant proteins in plants. Biotechnol Bioeng 103:562-573. doi: $10.1002 /$ bit.22278

Di Fiore S, Hoppmann V, Fischer R, Schillberg S (2004) Transient gene expression of recombinant terpenoid indole alkaloid enzymes in Catharanthus roseus leaves. Plant Mol Biol Report 22:15-22. doi: 10.1007/BF02773344

Dunoyer P, Himber C, Voinnet O (2006) Induction, suppression and requirement of RNA silencing pathways in virulent Agrobacterium tumefaciens infections. Nat Genet 38:258-263. doi: $10.1038 / \mathrm{ng} 1722$ 
Fang Y, Xie K, Xiong L (2014) Conserved miR164-targeted NAC genes negatively regulate drought resistance in rice. J Exp Bot 65:2119-2135. doi: 10.1093/jxb/eru072

Fernandes H, Konieczna M, Kolodziejczyk R, et al (2008) Crystallization and preliminary crystallographic studies of Hyp-1, a St John 's wort protein implicated in the biosynthesis of hypericin. Acta Crystallogr Sect F Struct Biol Cryst Commun 64:405-408. doi: $10.1107 /$ S1744309108009111

Fernandes H, Michalska K, Sikorski M, Jaskolski M (2013) Structural and functional aspects of PR-10 proteins. FEBS J 280:1169-1199. doi: 10.1111/febs.12114

Franklin G, Conceição LFR, Kombrink E, Dias ACP (2008) Hypericum perforatum plant cells reduce Agrobacterium viability during co-cultivation. Planta 227:1401-1408. doi: 10.1007/s00425-0080691-7

Franklin G, Concei ção LFR, Kombrink E, Dias ACP (2009) Xanthone biosynthesis in Hypericum perforatum cells provides antioxidant and antimicrobial protection upon biotic stress. Phytochemistry 70:60-68. doi: 10.1016/j.phytochem.2008.10.016

Fu J, Wang S (2011) Insights into Auxin Signaling in Plant -Pathogen Interactions. Front Plant Sci 2:1-7. doi: 10.3389/fpls.2011.00074

Gasic K, Hernandez A, Korban SS (2004) RNA extraction from different apple tissues rich in polyphenols and polysaccharides for cDNA library construction. Plant Mol Biol Report 22:437-438. doi: 10.1007/BF02772687

Gohlke J, Deeken R (2014) Plant responses to Agrobacterium tumefaciens and crown gall development. Front Plant Sci 5:1-11. doi: 10.3389/fpls.2014.00155

Gómez-Gómez L, Rubio-Moraga A, Ahrazem O (2011) Molecular cloning and characterisation of a pathogenesis-related protein CsPR10 from Crocus sativus . Plant Biol 13:297-303. doi: 10.1111/j.1438-8677.2010.00359.x

Goyal S, Lambert C, Cluzet S, M érillon JM, Ramawat KG (2012) Secondary metabolites and plant defence. In: M érillon J., Ramawat K. (eds) Plant defence: Biological control. progress in biological control. Springer, Dordrecht , pp 109-138

Guo H, Xie Q, Fei J, Chua N (2005) microRNA164 directs NAC1 mRNA cleavage to downregulate auxin signals for lateral root development. Plant Cell 1-12. doi: 10.1105/tpc.105.030841.lism

He M, Xu Y, Cao J, et al (2013) Subcellular localization and functional analyses of a PR10 protein gene from Vitis pseudoreticulata in response to Plasmopara viticola infection. Protoplasma 250:129-140. doi: 10.1007/s00709-012-0384-8 
Heldt H-W, Piechulla B (2011) Phenylpropanoids comprise a multitude of plant secondary metabolites and cell wall components. Plant Biochem 431-449. doi: 10.1016/B978-0-12-384986$1.00018-1$

Horsch RB, Fry JE, Hoffmann NL, et al (1985) A simple and general method for hybridization revealed the expected. Science 227:1229-1231. doi: 10.1126/science.227.4691.1229

Hou W N, Shakya P, Franklin G (2016) A perspective on Hypericum perforatum genetic transformation. Front Plant Sci 7:1-12. doi: 10.3389/fpls.2016.00879

Hou WN, Duan CG, Fang RX, et al (2011) Satellite RNA reduces expression of the 2b suppressor protein resulting in the attenuation of symptoms caused by Cucumber mosaic virus infection. Mol Plant Pathol 12:595-605. doi: 10.1111/j.1364-3703.2010.00696.x

Hsu SW, Liu MC, Zen KC, Wang CS (2014) Identification of the tapetum/microspore-specific promoter of the pathogenesis-related 10 gene and its regulation in the anther of Lilium longiflorum. Plant Sci 215-216:124-133. doi: 10.1016/j.plantsci.2013.11.006

Jiang $\mathrm{L}, \mathrm{Wu} \mathrm{J}$, Fan $\mathrm{S}$, et al (2015) Isolation and characterization of a novel pathogenesis-related protein gene (GmPRP) with induced expression in soybean (Glycine max) during infection with Phytophthora sojae. PLoS One 10:e0129932. doi: 10.1371/journal.pone.0129932

Karppinen K (2010) Biosynthesis of hypericins and hyperforins in Hypericum perforatum L.(St. John's wort) — precursors and genes involved. Dissertation, University of Oulu

Karppinen K, Derzs ó E, Jaakola L, Hohtola A (2016) Molecular cloning and expression analysis of hyp-1 type PR-10 family genes in Hypericum perforatum . Front Plant Sci 7:526. doi: $10.3389 /$ fpls.2016.00526

Kim SG, Kim ST, Wang Y, et al (2011) The RNase activity of rice probenazole-induced protein1 (PBZ1) plays a key role in cell death in plants. Mol Cells 31:25-31. doi: 10.1007/s10059-0110004-z

Kim ST, Yu S, Kang YH, et al (2008) The rice pathogen-related protein 10 (JIOsPR10) is induced by abiotic and biotic stresses and exhibits ribonuclease activity. Plant Cell Rep 27:593-603. doi: 10.1007/s00299-007-0485-6

Kondou Y, Higuchi M, Matsui M (2010) High- throughput characterization of plant gene functions by using gain-of-function technology. Annu Rev Plant Biol 61:373-393. doi: 10.1146/annurevarplant-042809-112143

Košuth J, Hrehorov á D, Jaskolski M, Čellárová E (2013) Stress-induced expression and structure of the putative gene hyp-1 for hypericin biosynthesis. Plant Cell Tissue Organ Cult 114:207-216. 
doi: 10.1007/s11240-013-0316-0

Košuth J, Katkov činová Z, Olexov á P, Čellárová E (2007) Expression of the hyp-1 gene in early stages of development of Hypericum perforatum L. Plant Cell Rep 26:211-217. doi: 10.1007/s00299-006-0240-4

Košuth J, Smelcerovic A, Borsch T, et al (2011) The hyp-1 gene is not a limiting factor for hypericin biosynthesis in the genus Hypericum. Funct Plant Biol 38:35-43. doi: 10.1071/FP10144

Krishnaswamy S, Baral PK, James MNG, Kav NNV (2011) Site-directed mutagenesis of histidine 69 and glutamic acid 148 alters the ribonuclease activity of pea ABR17 (PR10.4). Plant Physiol Biochem 49:958-962. doi: 10.1016/j.plaphy.2010.10.010

Lee MH, Jeon HS, Kim HG, Park OK (2017) An Arabidopsis NAC transcription factor NAC4 promotes pathogen-induced cell death under negative regulation by microRNA164. New Phytol 214:343-360. doi: 10.1111/nph.14371

Liscombe DK, O 'Connor SE (2011) A virus-induced gene silencing approach to understanding alkaloid metabolism in Catharanthus roseus . Phytochemistry 72:1969-1977. doi: 10.1016/j.phytochem.2011.07.001

Mazid M, Khan TA, Mohammad F (2011) Role of secondary metabolites in defense mechanisms of plants. Biol Med 3:232-249. doi: citeulike-article-id:10435815

Michalska K, Fernandes H, Sikorski M, Jaskolski M (2010) Crystal structure of Hyp-1, a St. John 's wort protein implicated in the biosynthesis of hypericin. J Struct Biol 169:161-171. doi: 10.1016/j.jsb.2009.10.008

Miedes E, Vanholme R, Boerjan W, Molina A (2014) The role of the secondary cell wall in plant resistance to pathogens. Front Plant Sci 5:1-14. doi: 10.3389/fpls.2014.00358

Nelson BK, Cai X, Nebenf ühr A (2007) A multicolored set of in vivo organelle markers for colocalization studies in Arabidopsis and other plants. Plant J 51:1126-1136. doi: 10.1111/j.1365313X.2007.03212.x

Park CJ, Kim KJ, Shin R, et al (2004) Pathogenesis-related protein 10 isolated from hot pepper functions as a ribonuclease in an antiviral pathway. Plant J 37:186-198. doi: 10.1046/j.1365313X.2003.01951.x

Patočka J (2003) The chemistry, pharmacology, and toxicology of the biologically active constituents of the herb Hypericum perforatum L. J Appl Biomed 1:61-70

Pinweha N, Asvarak T, Viboonjun U, Narangajavana J (2015) Involvement of miR160/miR393 and 
their targets in cassava responses to anthracnose disease. J Plant Physiol 174:26-35. doi: 10.1016/j.jplph.2014.09.006

Pitzschke A (2013) Agrobacterium infection and plant defense - transformation success hangs by a thread. Front Plant Sci 4:1-12. doi: 10.3389/fpls.2013.00519

Pruss GJ, Nester EW, Vance V (2008) Infiltration with Agrobacterium tumefaciens induces host defense and development-dependent responses in the infiltrated zone. Mol Plant Microbe Interact 21:1528-1538. doi: 10.1094/MPMI-21-12-1528

Pungartnik C, da Silva AC, de Melo SA, et al (2009) High- affinity copper transport and Snq2 export permease of Saccharomyces cerevisiae modulate cytotoxicity of PR-10 from Theobroma cacao. Mol Plant-Microbe Interact 22:39-51. doi: 10.1094/MPMI-22-1-0039

Saddiqe Z, Naeem I, Maimoona A (2010) A review of the antibacterial activity of Hypericum perforatum L. J Ethnopharmacol 131:511-521. doi: 10.1016/j.jep.2010.07.034

Schindelin J, Arganda-Carreras I, Frise E, et al (2012) Fiji: An open-source platform for biologicalimage analysis. Nat Methods 9:676-682. doi: 10.1038/nmeth.2019

Schmidt GW, Delaney SK (2010) Stable internal reference genes for normalization of real-time RTPCR in tobacco ( Nicotiana tabacum ) during development and abiotic stress. Mol Genet Genomics 283:233-241. doi: 10.1007/s00438-010-0511-1

Sheshadri SA, Nishanth MJ, Yamine V, Simon B (2018) Effect of Melatonin on the stability and expression of reference genes in Catharanthus roseus. Sci Rep 1-11. doi: 10.1038/s41598-01820474-2

Shi R, Sun YH, Zhang XH, Chiang VL (2012) Poly(T) adaptor RT-PCR. In: Fan JB. (eds) Nextgeneration microRNA expression profiling technology. Methods in Molecular Biology (Methods and Protocols). Humana Press, New York, pp 53-66

Shi R, Yang C, Lu S, et al (2010) Specific down-regulation of $P A L$ genes by artificial microRNAs in Populus trichocarpa. Planta 232:1281-1288. doi: 10.1007/s00425-010-1253-3

Singh RK, Hou WN , Franklin G (2016) Construction of hypericin gland-specific cDNA library via suppression subtractive hybridization. In: Jain S. (eds) Protocols for In Vitro Cultures and Secondary Metabolite Analysis of Aromatic and Medicinal Plants, Second Edition. Methods in Molecular Biology. Humana Press, New York, pp 317-334

Sliwiak J, Dauter Z, Jaskolski M (2016) Crystal structure of Hyp-1, a Hypericum perforatum PR-10 protein, in complex with Melatonin. Front Plant Sci 7:1-10. doi: 10.3389/fpls.2016.00668 
Tamura K, Stecher G, Peterson D, et al (2013) MEGA6: Molecular evolutionary genetics analysis version 6.0. Mol Biol Evol 30:2725-2729. doi: 10.1093/molbev/mst197

Tatsis EC, Boeren S, Exarchou V, et al (2007) Identification of the major constituents of Hypericum perforatum by LC/SPE/NMR and/or LC/MS. Phytochemistry 68:383-393. doi: 10.1016/j.phytochem.2006.11.026

Wójcik AM, Nodine MD, Gaj MD (2017) miR160 and miR166/165 contribute to the LEC2-mediated auxin response involved in the somatic embryogenesis induction in Arabidopsis. Front Plant Sci 8:1-17. doi: 10.3389/fpls.2017.02024

Xie YR, Chen ZY, Brown RL, Bhatnagar D (2010) Expression and functional characterization of two pathogenesis-related protein 10 genes from Zea mays . J Plant Physiol 167:121-130. doi: 10.1016/j.jplph.2009.07.004

Xu P, Jiang L, Wu J, et al (2014) Isolation and characterization of a pathogenesis-related protein 10 gene ( GmPR10) with induced expression in soybean ( Glycine max ) during infection with Phytophthora sojae. Mol Biol Rep 41:4899-4909. doi: 10.1007/s11033-014-3356-6

Zhang L, Yao L, Zhang N, et al (2018) Lateral root development in potato is mediated by Stu-mi164 regulation of NAC transcription factor. Front Plant Sci 9:383. doi: 10.3389/fpls.2018.00383

Zhang R, Wang Y, Liu G, Li H (2010) Cloning and characterization of a pathogenesis-related gene (ThPR10) from Tamarix hispida . Acta Biol Cracoviensia Ser Bot 52:17-25. doi: 10.2478/v10182-010-0019-3

\section{Figure legends}

Fig. 1 Detection of Agrobacterium-mediated expression in GFP-Hyp-1 tobacco T2 lines by GUS staining. (a) Wild-type (WT) plant inoculated with A. tumefaciens EHA105 containing the empty vector as negative control. (b) WT plant inoculated with A. tumefaciens containing pCAMBIA 2301GUS as positive control. (c) GFP transgenic line inoculated with A. tumefaciens containing pCAMBIA 2301-GUS as positive control. (d) GFP-Hyp-1-3-2 and (e) GFP-Hyp-1-3-3 transgenic lines inoculated with A. tumefaciens containing pCAMBIA 2301-GUS. (f) GFP-Hyp-1-4-1 and (g) GFP-Hyp-1-4-3 transgenic lines inoculated with A. tumefaciens containing pCAMBIA 2301-GUS. (h) GFP-Hyp-1-5-2 and (i) GFP-Hyp-1-5-3 transgenic lines inoculated with A. tumefaciens containing pCAMBIA 2301-GUS. Bars in each panel represent $10 \mathrm{~mm}$

Fig. 2 Detection of Agrobacterium-mediated expression in Hyp-1 L. sativa BL T1 lines by GUS staining. (a) Wild-type (WT) plant inoculated with A.tumefaciens EHA105 containing the empty vector as negative control. (b) WT plant inoculated with A. tumefaciens containing pCAMBIA 2301- 
GUS as positive control. ( c-d) GFP-Hyp-1-1/-3 transgenic lines inoculated with A. tumefaciens containing GUS vector. ( e-f) Hyp-1-6/-8 transgenic lines inoculated with A. tumefaciens containing GUS vector. Bars in each panel represent $10 \mathrm{~mm}$

Fig. 3 Detection of Agrobacterium-mediated expression in Hyp-1 L. sativa SBR T1 lines by GUS staining. (a) Wild-type (WT) plant inoculated with A.tumefaciens EHA105 containing the empty vector as negative control ( b) WT plant inoculated with A. tumefaciens containing pCAMBIA 2301GUS as positive control. (c) Hyp-1-12 transgenic line inoculated with A. tumefaciens containing GUS vector. Bars in each panel represent $10 \mathrm{~mm}$

Fig. 4 Detection of Agrobacterium-mediated expression in GFP-Hyp-N tobacco T2 lines by GUS staining. (a) Wild-type (WT) plant inoculated with A. tumefaciens containing the empty vector as negative control. (b) WT plant inoculated with A. tumefaciens containing pCAMBIA 2301-GUS as positive control. ( c-e) GFP-Hyp-N-2 (1/-2/-3) transgenic lines inoculated with A. tumefaciens containing GUS vector. (f-h) GFP-Hyp-N-3 (1/-2/-3) transgenic lines inoculated with A. tumefaciens containing GUS vector. Bars in each panel represent $10 \mathrm{~mm}$

Fig. 5 Subcellular localization of GFP-Hyp-1 at the plasma membrane, nucleus and cytoplasm. Leaves of N. tabacum were co-infiltrated with GFP-Hyp-1 and plasma membrane marker PM-RK. Panels show either monochrome green (from GFP) ( $\mathbf{a}$ and $\mathbf{e}$ ) or red (from mCherry) (b and $\mathbf{f}$ ) fluorescence, overlays for colocalization studies ( $\mathbf{c}$ and $\mathbf{g}$ ), or bright field ( $\mathbf{d}$ and $\mathbf{h}$ ). Arrows indicate nucleus (N). Scale bars in all images are $25 \mu \mathrm{m}$

Fig. 6 Fluorescence microscopy distinguish the localizati on of GFP-Hyp-1 between cell w all and plasma membrane. (a-f) GFP transient expression in leaf epidermal cells of $\quad$. tabacum. (g-o) GFPHyp-1 transient expression in leaf epidermal cells of $\quad$. tabacum. (a-c, g-i) Leaf explants incubated with $\mathrm{H}_{2} \mathrm{O}$ prior to observation . (d-f, i-o) L eaf explants incubated with $1 \mathrm{M}$ mannitol prior to observation. The arrows show the plasma membrane section detached from the cell wall. Scale bars in all images are $25 \mu \mathrm{m}$

Fig. 7 Confocal fluorescence microscopy and western blot analysis in GFP-Hyp-1 transgenic lines. (a) GFP transient expression in leaf epidermal cells of $\quad N$. benthamiana. (b) Leaf epidermal cells of $N$. tabacum GFP-Hyp-1-4 transgenic line. (c-d) Leaf epidermal cells of N. tabacum GFP-Hyp-1-5 transgenic line. Contrary to the transient expression of free GFP, constitutive fluorescence in GFPHyp-1 transgenic plants were also detected in stomata guard cells (d). (e) Western blot to detect GFPHyp-1 expression by using antibodies against GFP. GFP+ was free GFP agroinfiltrated into $N$. benthamiana as positive control. Lane M shows the molecular weight markers in kilodalton ( $\mathrm{kDa})$. The panel below the western blot shows the membrane stained with Ponceau-S after blotting as control of loading. Arrows indicate nucleus (N) and stomata (S). Scale bars in all images are $25 \mu \mathrm{m}$ 
Fig. 8 Quantitative real time PCR to assess core genes involved in different plant defense pathways in GFP-Hyp-1 transgenic tobacco plants. (a) Relative transcript levels of Hyp-1. (b-c) Relative transcript levels of TIRI and ARF8. (d) Relative transcript levels of $L O X$. (e-f) Relative transcript levels of PR10 and PR-1. (g-j) Relative transcript levels of $A G O 1$, MiR160, MiR164ab and MiR164c. ( k-n) Relative transcript levels of PAL1, PAL4,CCD and CAD. (k, l-o) Relative transcript levels of PAL1, $P A L 4$, and CHS. (p-r) Relative transcript levels of $A P X, C A T$, and $F e-S O D$. Asterisks denote the significance levels compared with control: *, $P \pi 0.05$; **, $P \pi 0.01$; ***, $P \pi 0.001$; ****, $\mathrm{P} \pi 0.0001$ and ns denotes not significant

Fig. 9 Silencing of $C r I P R$ in C.roseus results in decreased Agrobacterium-mediated expression efficiency. (a) Representative C.roseus plants inoculated with Agrobacterium strains containing pTRV2: CrChlH, pTRV2:00 and pTRV2: CrIPR at 21 days after inoculation (dpi). ( b) Silencing of CrIPR transcripts was analyzed by semiquantitative RT-PCR at $21 \mathrm{dpi}$. The same RT reactions were used to amplify $C r R P S 9$ gene transcripts as a control. ( c) Western blot analysis of GFP accumulation in $C r I P R$-silenced and control C. roseus leaves at 3 and 5 days after infiltration with an Agrobacterium strain expressing GFP. The panel below the western blot shows the membrane stained with Ponceau-S after blotting as control of loading. Lane $\mathrm{M}$, molecular weight markers. ( Expression analysis of $C r P R-1$ and $C r P R-5$ in $C r I P R$-silenced and control C. roseus leaves at 21 dpi. CrRPS 9 was used as reference gene. *, $P \pi 0.05$; ns, not significant

\section{Supplementary data}

Table S1 Gene-specific primers use d for cloning, VIGS and gene expression analysis by real-time quantitative reverse transcriptase PCR

Table S2 Putative functional classification of transcripts identified by BLASTX analysis from two forward subtractive cDNA libraries from Agrobacterium tumefaciens-treated Hypericum perforatum L. suspension cells (12hr and 24hr)

Fig. S1 Generation of Nicotiana tabacum GFP-Hyp-1 overexpressing plants. (a) Wild-type $N$. tabacum control plant. ( b) GFP transgenic plant as control. ( c-f) GFP-Hyp-1-2/-3/-4/-5 transgenic plant lines. ( g) Schematic diagram of pBI121-GFP-Hyp-1 made by software Vector NTI. Confirmation of T-DNA insertion by RT-PCR

Fig. S2 Generation of L. sativa BL Hyp- 1 overexpression plants. (a, d) Wild-type L. sativa BL control plant. (b, e) BL GFP-Hyp-1 transgenic plant. (c, f) BL Hyp-1 transgenic plant

Fig. S3 Generation of L. sativa SBR Hyp-1 overexpression plants. (a, c) Wild-type L. sativa SBR control plant. (b, d) SBR Hyp-1 transgenic plant

Fig. S4 Schematic diagram of pBI121-GFP-Hyp-1-N made by software Vector NTI. The cDNA 
sequence encoding the N-terminal 80 amino acids of Hyp-1 (yellow arrow) followed by two stop codons was fused to GFP (orange arrow). The characteristic Bet.v.1 domain (89-120 aa residues) missing in GFP-Hyp-1 is showed by a black arrow marked with a red cross

Fig. S5 Phylogenetic relationships of Hyp-1 homologs in plants, constructed using the program MEGA 6. The amino acid sequences were obtained from GenBank. Plant Latin names and GenBank accession numbers were used to mark the phylogenetic tree. Hyp- 1 was framed in red box and the VIGS candidate from Catharanthus roseus was framed in yellow box. The scale was shown in units of number of amino acid substitutions per site 


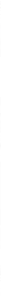


8 두웅 10.7의 360 


\section{GFP-Hyp-1}
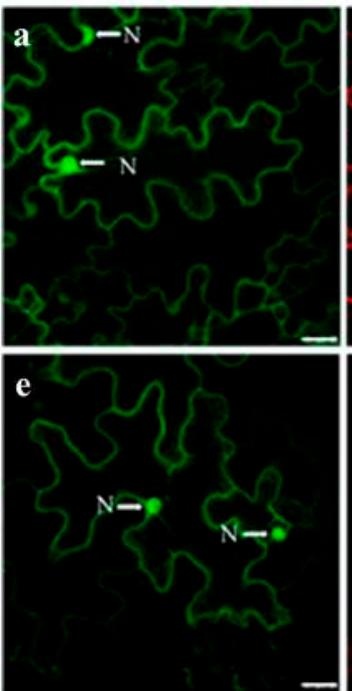

PM-RK
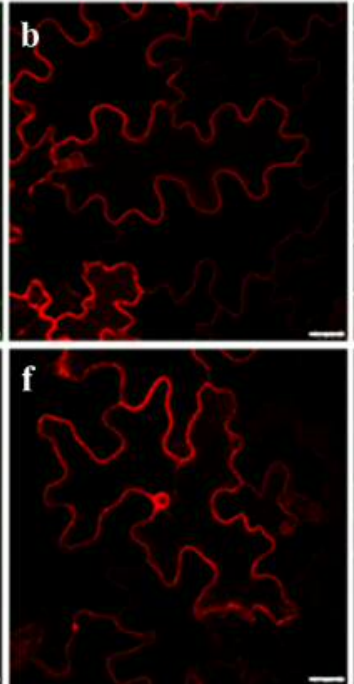

Merge

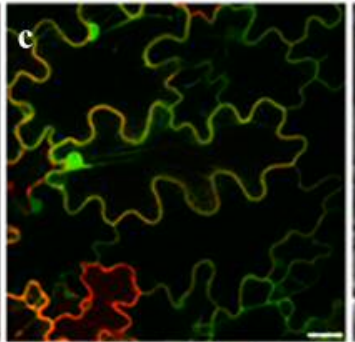

Bright field

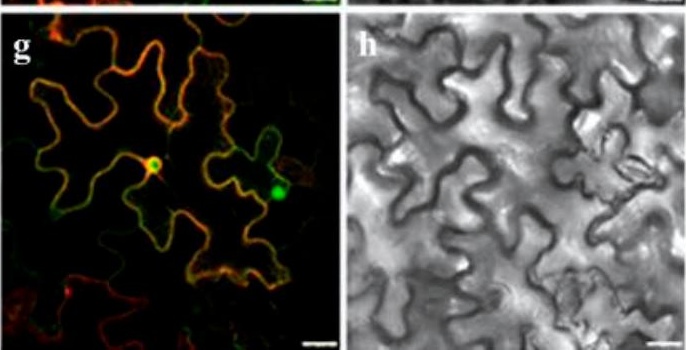


GFP +

$\mathrm{H}_{2} \mathrm{O}$
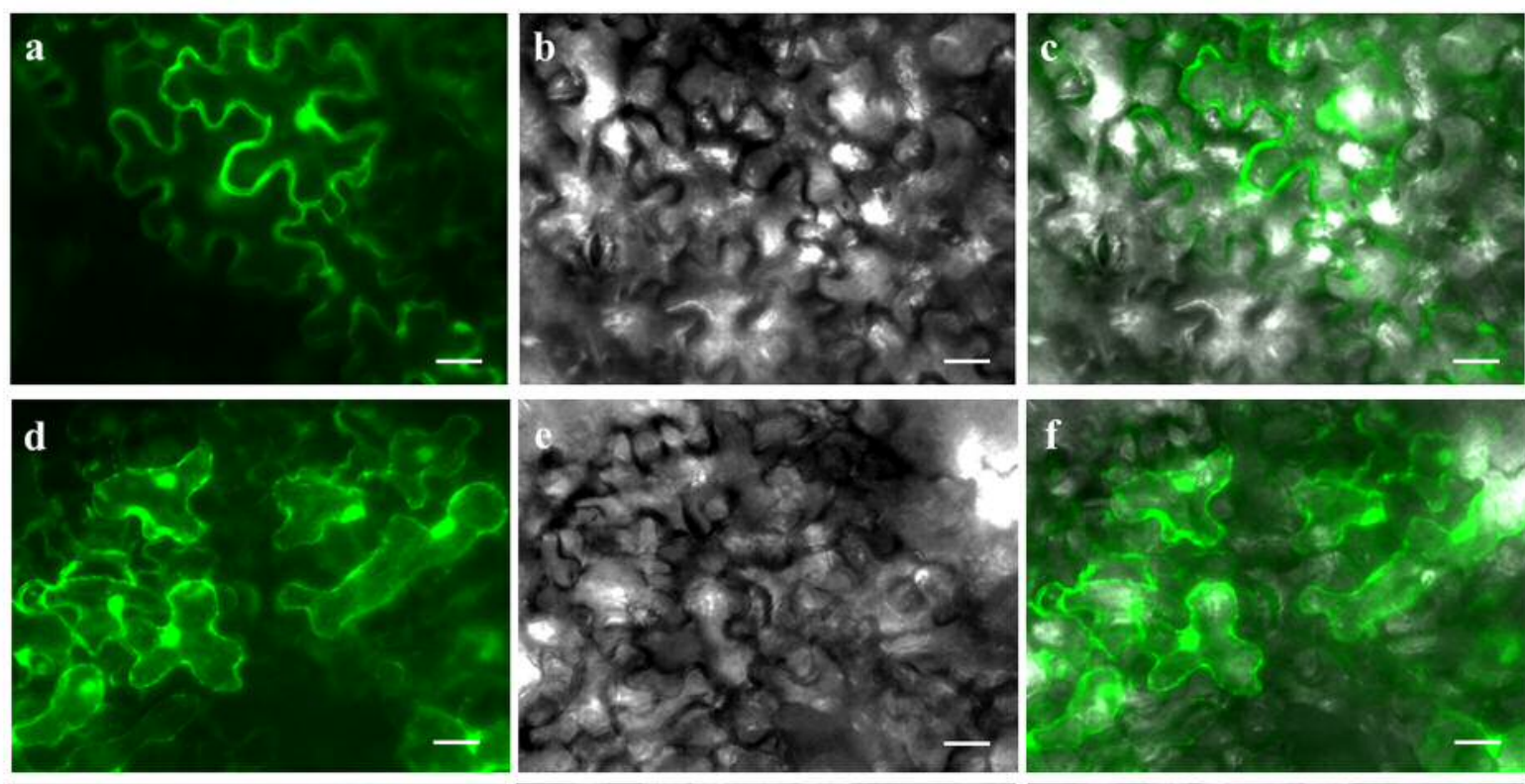

$-$
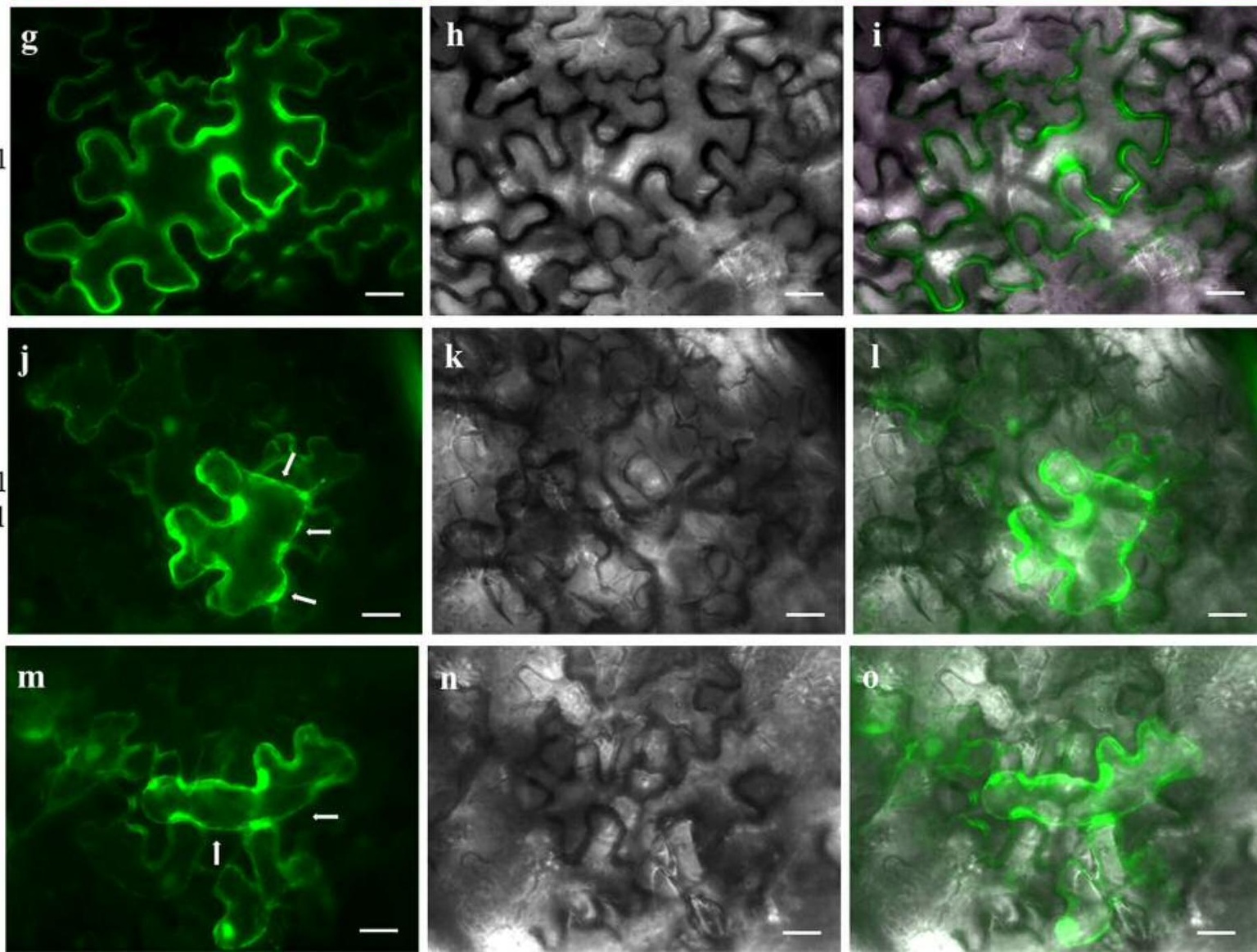


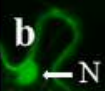

$$
\mathrm{N}-
$$

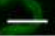

c

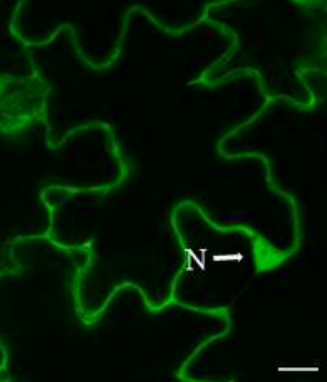

d

$\mathrm{S}-$

$-\mathrm{N}$

GFP $+\quad M \quad$ GFP-Hyp-1-4 GFP-Hyp-1-5

GFP-Hyp-1

GFP 
a

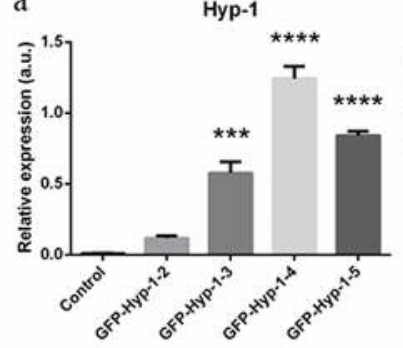

d

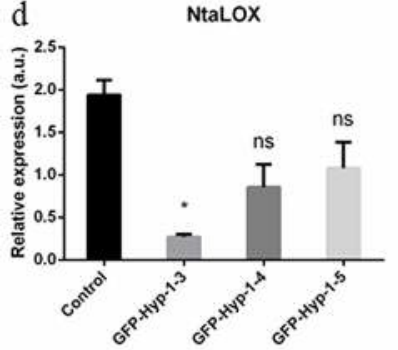

$\mathrm{g}$

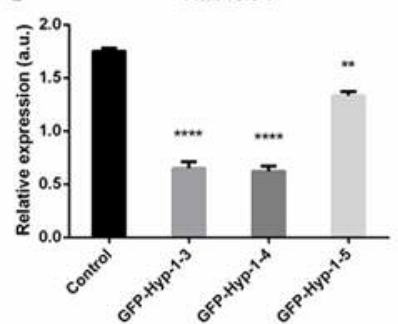

j

\section{음}

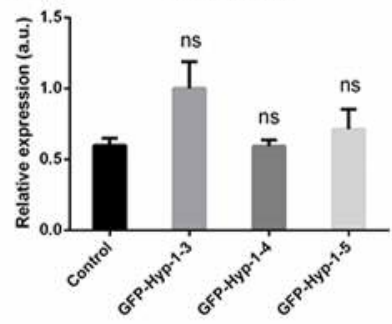

m

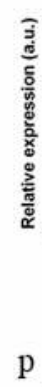

NtaCCR
- Control

- GFP-Hyp-1-3

- GFP-Hyp-1-4

GFP-Hyp-1-5 b

- Control

- GFP-Hyp-1-3

- GFP-Hyp-1-4

- GFP-Hyp-1-5
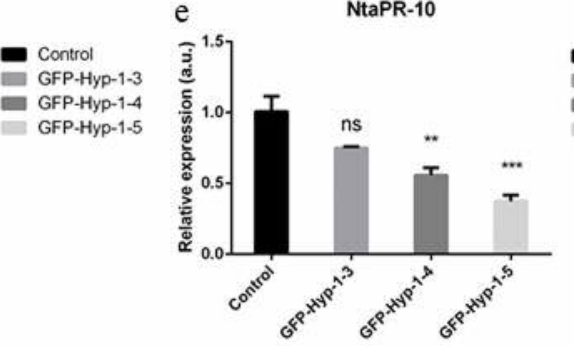

h
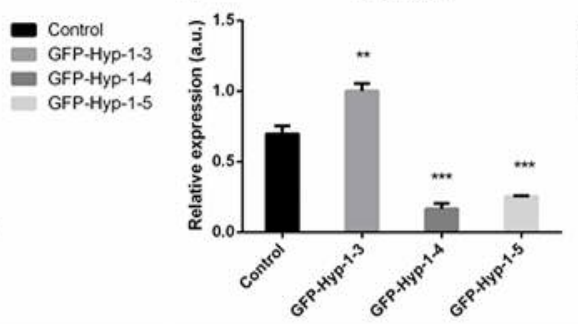

k

- Control

- GFP-Hyp-1-4

EFP.Hyp-1.5

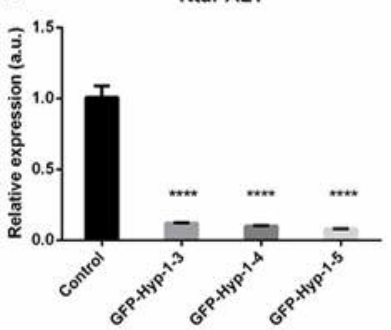

n

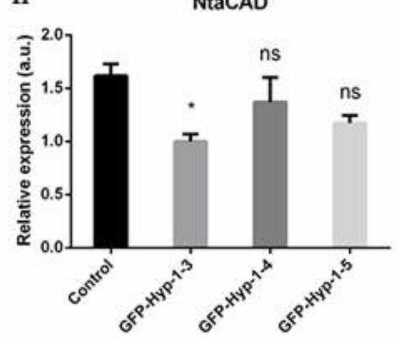

q
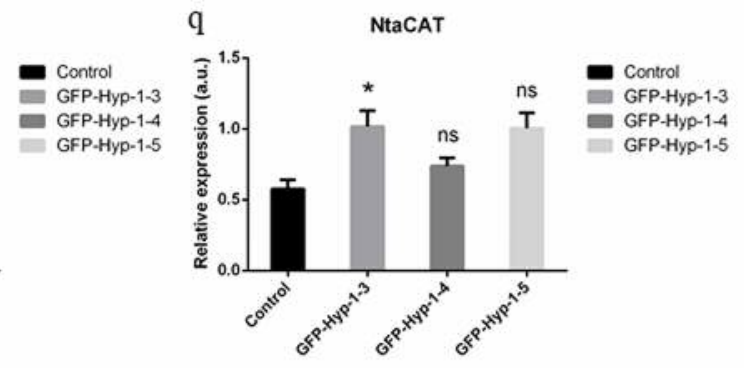

c

NtaARF8

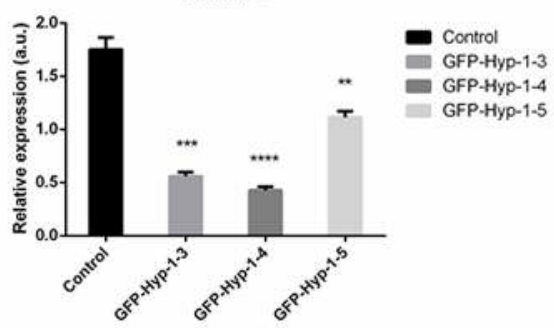

f

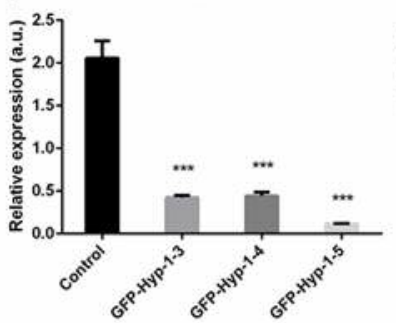

- Control

GFP.Hyp-1-3

GFP-Hyp-1-4

GFP-Hyp-1-5
- Control

- GFP.Hyp-1-3

- GFP.Hyp-1-4

GFP-Hyp-1-5

i NtaMiR164ab

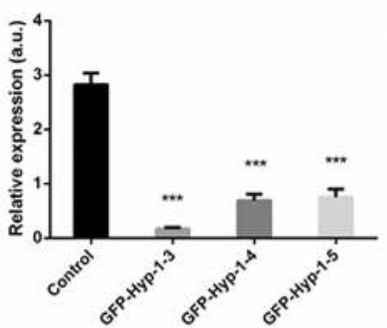

1

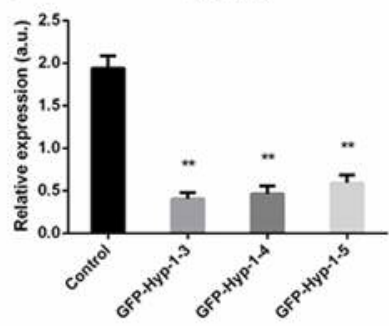

0

- Control - GFP.Hyp-1-4 - GFP.Hyp-1-5

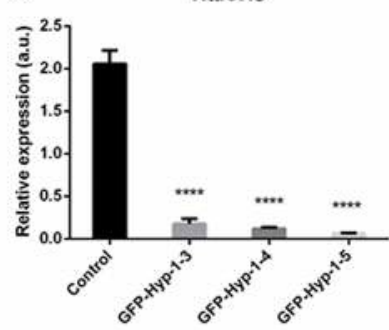

I

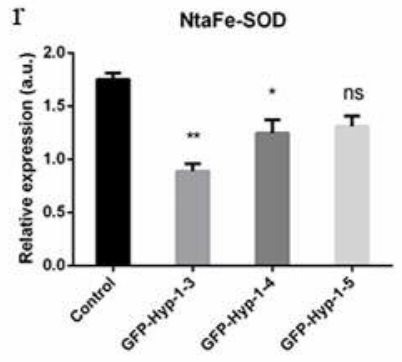

- Control

GFP-Hyp-1-3

GFP.Hyp-1-4

GFP-Hyp-1-5
- Control

- GFP.Hyp-1-3

III GFP-Hyp-1-4

GFP-Hyp-1-5
- Control

EFP.Hyp-1-3

- GFP-Hyp-1-4

GFP.Hyp-1-5

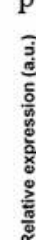

$\mathrm{p}$

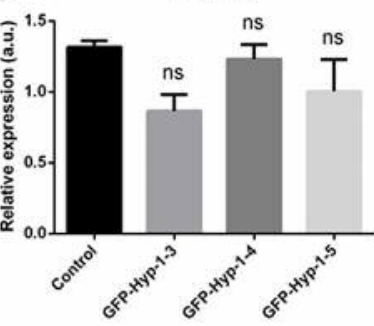



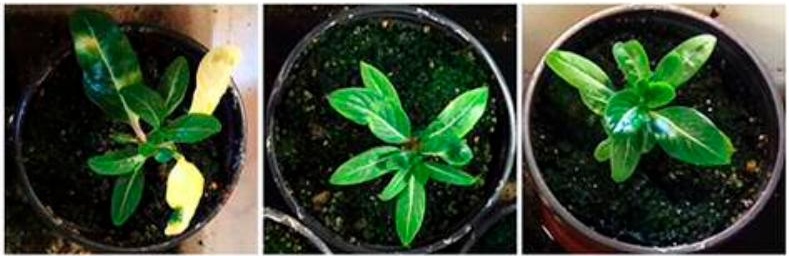

b

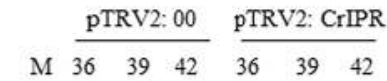

CrIPR

$200 \mathrm{bp}$

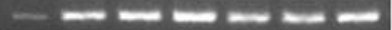

CrRSP9

c

$3 \mathrm{dpi}$

5 dpi

$\begin{array}{llll}\text { pTRV2:00 pTRV2:CrIPR } & \text { pTRV2:00 pTRV2:CrIPR }\end{array}$

GFP

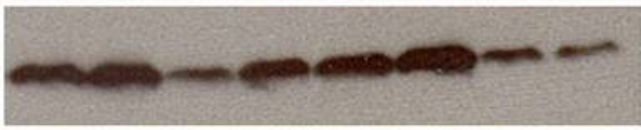

d

CrPR-1

CrPR.5
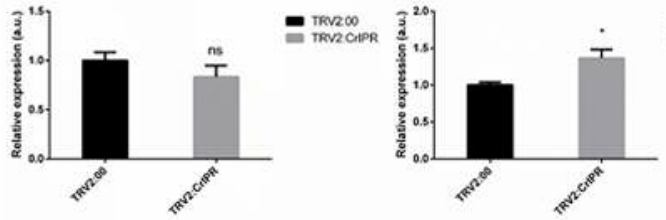

- TRV 200

THV CIPR 
Transgenic expression of Hyp-1 gene from Hypericum perforatum L. alters expression of defense related genes and modulates tolerance to Agrobacterium tumefaciens

Weina $\mathrm{Hou}^{1}$, Rupesh Kumar Singh ${ }^{2}$, Pan $\mathrm{Zhao}^{3}$, Viviana Martins ${ }^{4}$, Emmanuel Aguilar ${ }^{5}$, Tomás Canto $^{5}$, Francisco Tenllado ${ }^{5 *}$, Alberto Carlos Pires Dias ${ }^{1,4,6}$ Supplemental Data 


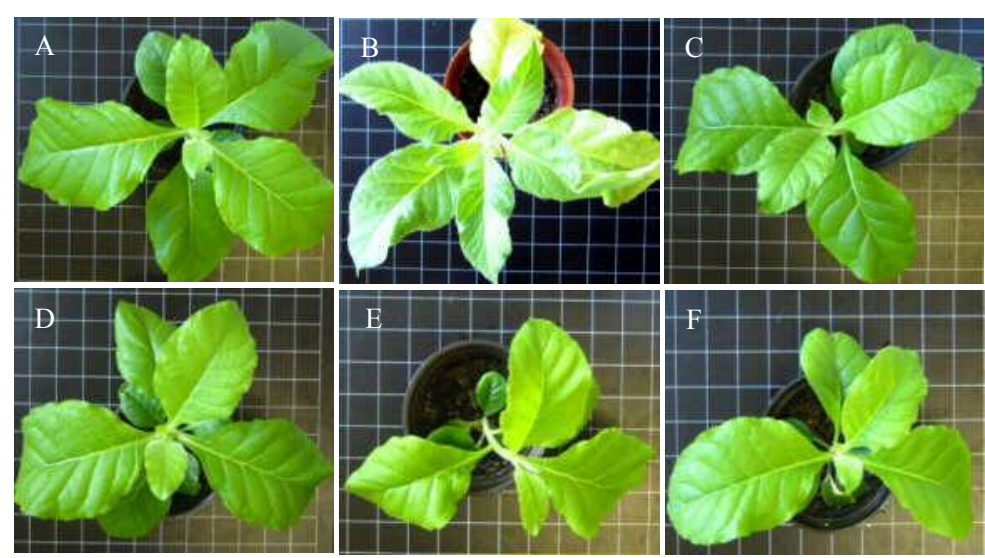

G NOS-P

GFP-Hyp-1

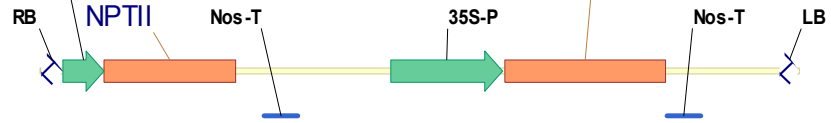

pBI121-GFP-Hyp-1

GFP

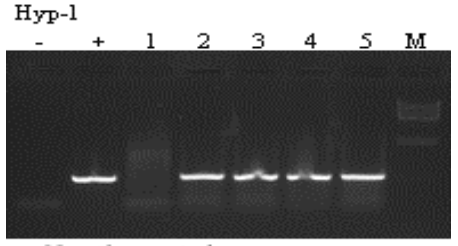

Negative control

+. Positive control (Hyp-1 PCR product)

1. NT WT

+. Positive control (GFP PCR product) 1. NT WT

2-5. GTP-Hyp-1-2/-3/-4/-5

M. DNA Inp-1-2/-3/-4/-5

Fig S1. Generation of Nicotiana tabacum GFP-Hyp-1 overexpressing plants. (A) Wild-type $N$. tabacum control plant. (B) GFP transgenic plant as control. (C-F) GFP-Hyp-1-2/-3/-4/-5 transgenic plant lines. (G) Schematic diagram of pBI121-GFP-Hyp-1 made by software Vector NTI. (H) Confirmation of T-DNA insertion by RT-PCR. 


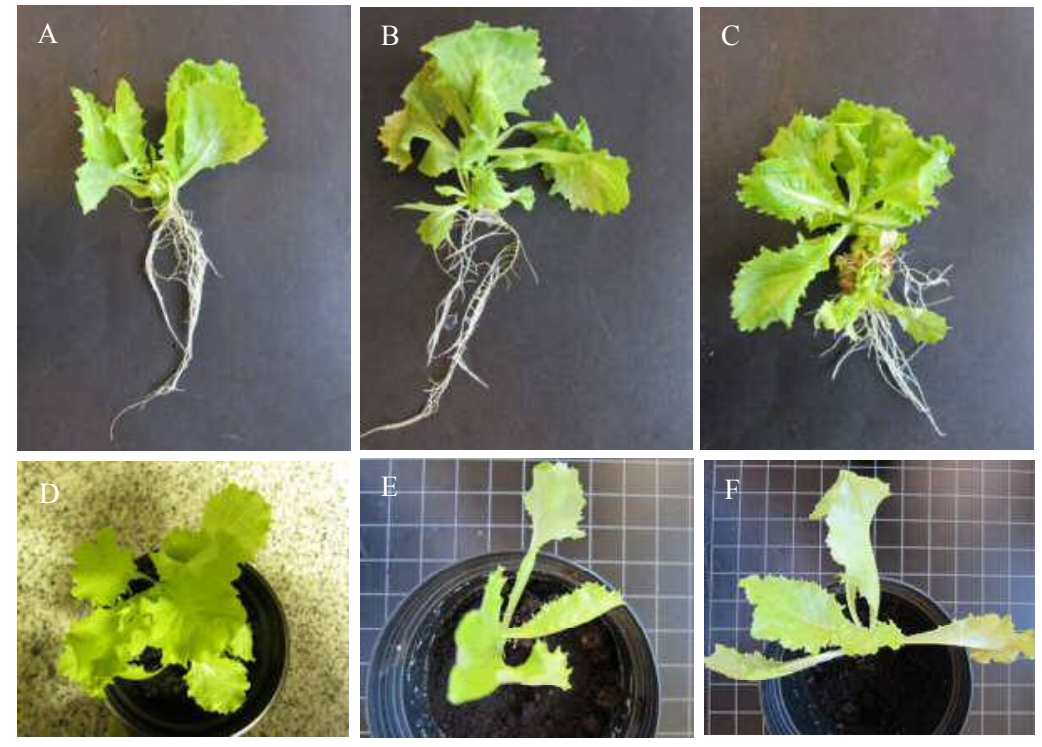

Fig S2. Generation of L. sativa BL Hyp-1 overexpression plants. (A, D) Wild-type $L$. sativa BL control plant. (B, E) BL GFP-Hyp-1 transgenic plant. (C, F) BL Hyp-1 transgenic plant. 


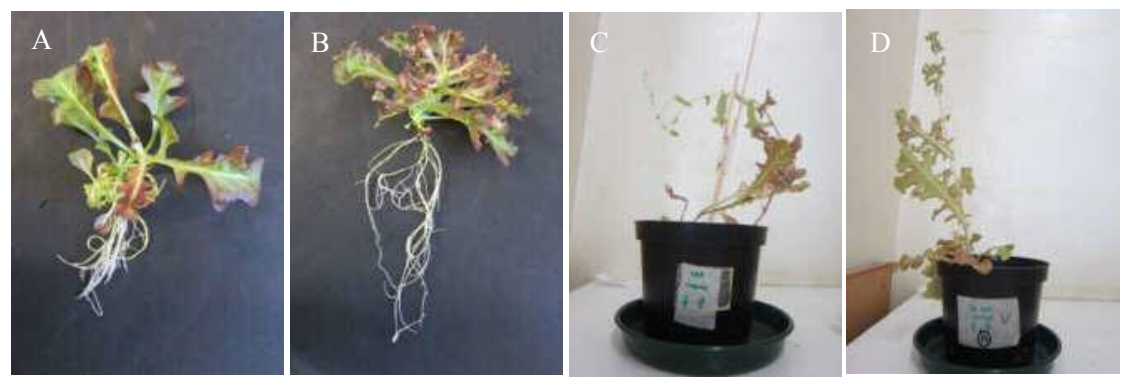

Fig S3. Generation of L. sativa SBR Hyp-1 overexpression plants. (A, C) Wild-type L. sativa SBR control plant. (B, D) SBR Hyp-1 transgenic plant. 


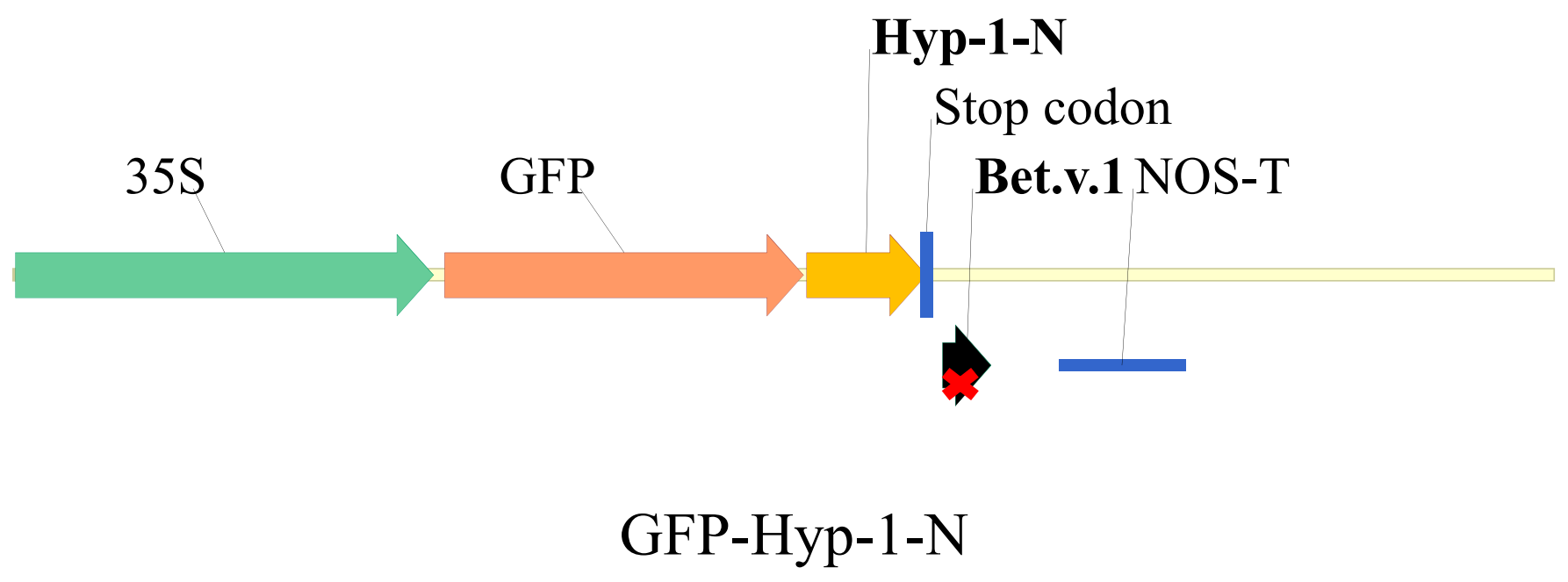

Fig S4. Schematic diagram of pBI121-GFP-Hyp-1-N made by software Vector NTI. The cDNA sequence encoding the N-terminal 80 amino acids of Hyp-1 (yellow arrow) followed by two stop codons was fused to GFP (orange arrow). The characteristic Bet.v.1 domain (89-120 aa residues) missing in GFP-Hyp-1 is showed by a black arrow marked with a red cross. 


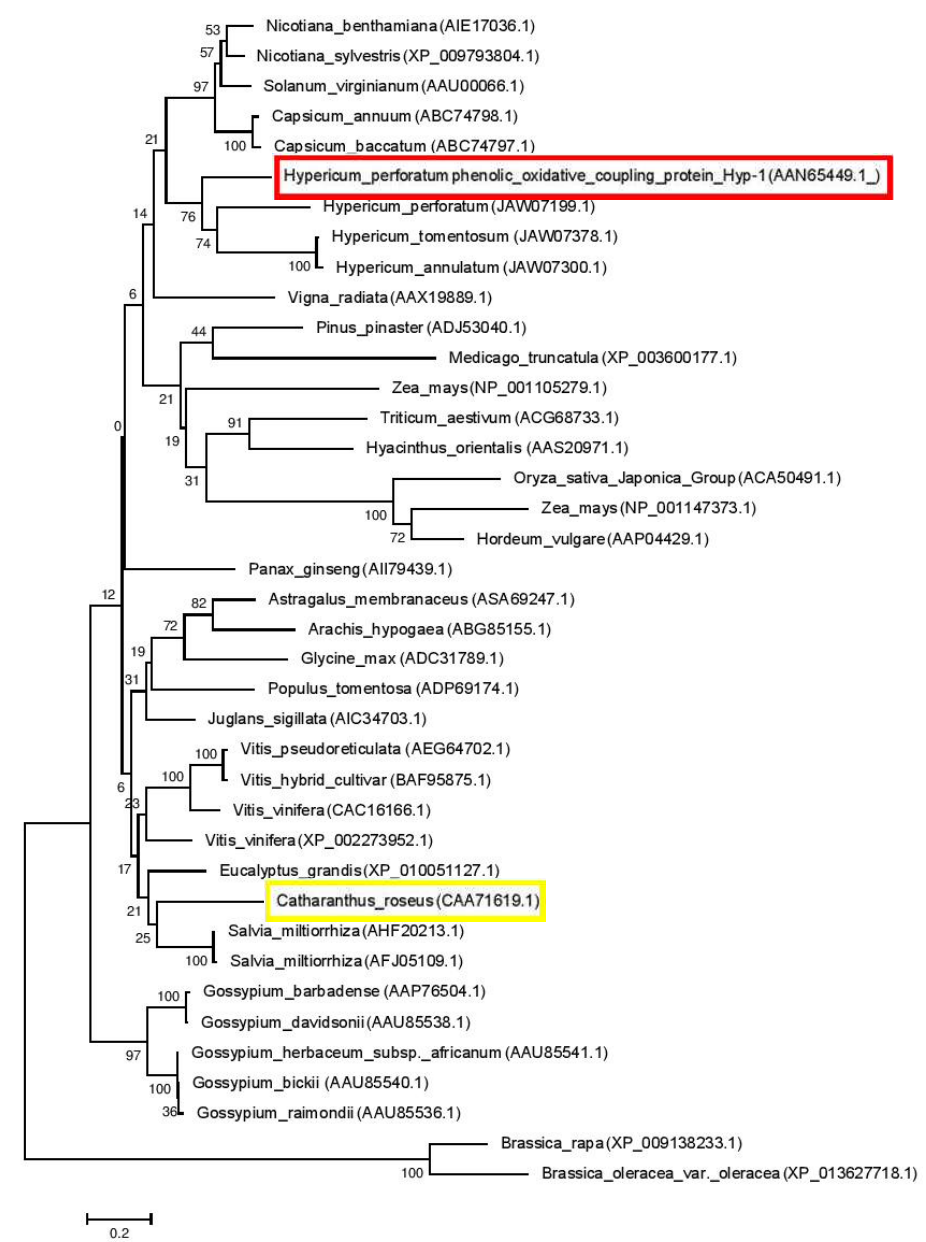

Fig S5. Phylogenetic relationships of Hyp-1 homologs in plants, constructed using the program MEGA 6. The amino acid sequences were obtained from GenBank. Plant Latin names and GenBank accession numbers were used to marker the phylogenetic tree. Hyp-1 was framed in red box and the VIGS candidate from C. roseus was framed in yellow box. The scale was shown in units of number of amino acid substitutions per site. 
Transgenic expression of Hyp-1 gene from Hypericum perforatum $\mathrm{L}$. alters expression of defense related genes and modulates tolerance to Agrobacterium tumefaciens

Weina Hou ${ }^{1}$, Rupesh Kumar Singh ${ }^{2}$, Pan Zhao ${ }^{3}$, Viviana Martins ${ }^{4}$, Emmanuel Aguilar $^{5}$, Tomás Canto ${ }^{5}$, Francisco Tenllado ${ }^{5}$, Alberto Carlos Pires Dias ${ }^{1,4,6}$

Supplemental Data

Table S1 Gene-specific primers used for cloning, VIGS and gene expression analysis by real-time quantitative reverse transcriptase PCR

\begin{tabular}{|c|c|}
\hline Gene & Primers (5'-3') \\
\hline \multicolumn{2}{|c|}{ Primers for plasmid construction } \\
\hline BamHI-GFP-F & Forward: GTGCACGGATCCATGGTGAGCAAGGGCGAG ${ }^{\mathrm{a}}$ \\
\hline GFP-SpeI-SacI-R & Reverse: CTGAGCTCACTAGTCTTGTACAGCTCGTCC \\
\hline SpeI-Hyp-1-F & Forward: GTGCACACT $\boldsymbol{A} \boldsymbol{G T A T G G C G G C G T A C A C T A T T}{ }^{\mathrm{c}}$ \\
\hline Hyp-1-SacI-R & Reverse: GTGCACGAGCTCTTAAGCGAAAACTTCAGG ${ }^{\mathrm{d}}$ \\
\hline STOP-Hyp-F & Forward: TAGTAATGATATTGCAAGTACAC \\
\hline STOP-Hyp-R & Reverse: GAAATTTGCGGCATCAATTTCAT \\
\hline \multicolumn{2}{|c|}{ Primers for RT-qPCR } \\
\hline \multirow[t]{2}{*}{ Hyp-1 } & Forward: GAACGCCTGGATTTAGTGGA \\
\hline & Reverse: AAGCTTGTAGGTGGCCGTAA \\
\hline \multirow[t]{2}{*}{ NtaTIR1 } & Forward: ACTCTTGGGCCGCTTGATACTG \\
\hline & Reverse: CGCCGCAATTCTTTACAGTTCTCC \\
\hline \multirow[t]{2}{*}{ NtaARF8 } & Forward: GCTGCCTGCTCAAACTCAAATGG \\
\hline & Reverse: GCTGATTGCCGACTTGTTGCTG \\
\hline \multirow[t]{2}{*}{ NtaLOX1 } & Forward: GCAGACACTGCTTGGCATTTCC \\
\hline & Reverse: TGCCCGAGGTAAAGTGTATCCG \\
\hline \multirow[t]{2}{*}{ NtaPR-10 } & Forward: CTTGCCAATTCTACCGTCTATGCC \\
\hline & Reverse: ATTAGCCGAGCTGGACGCAAAC \\
\hline \multirow[t]{2}{*}{ NtaPR-1 } & Forward: AGGCGTGGAACCATTAACTTGGG \\
\hline & Reverse: GCAGTCTGCAGCCAATTGAGAAAC \\
\hline \multirow[t]{2}{*}{ NtaAGO1 } & Forward: CATTTGGCAGCTTTCCGTGCTC \\
\hline & Reverse: TGCGCTTGTGACTGATCCACTG \\
\hline \multirow[t]{2}{*}{ NtaPAL1 } & Forward: ATCACTGCCTCGGGTGATCTTG \\
\hline & Reverse: GCCTTGGAATTAGGCCTACCAGTG \\
\hline \multirow[t]{2}{*}{ NtaPAL4 } & Forward: TGCCATCTAACCTCACCGCATC \\
\hline & Reverse: AGATGCCATGGCGATTTCAGCTC \\
\hline \multirow[t]{2}{*}{ NtaCCR } & Forward: AGAACAAGCAGCATGGGACGAG \\
\hline & Reverse: CACCAACACTGGGTTGATTGCC \\
\hline \multirow{2}{*}{ NtaCAD } & Forward: TGTGGTTGATGGGTGTGAAGGC \\
\hline & Reverse: TCTGCCTGTGGATCTTTGACTCC \\
\hline \multirow[t]{2}{*}{ NtaCHS } & Forward: CGCTCGAGTCCTTGTTGTTTGTTC \\
\hline & Reverse: AAGTGGGTGTCATTGGGTCCAC \\
\hline \multirow[t]{2}{*}{ NtaCAT } & Forward: ATGCAAAATCGTTCCCTGACA \\
\hline & Reverse: TCGAAAAACCAGGCAAAAGTA \\
\hline \multirow[t]{2}{*}{ NtaAPX } & Forward: TCAAGGCTGAGCAAGGACATGG \\
\hline & Reverse: TCCTTGATGGGCTCCAAGAGTC \\
\hline
\end{tabular}




\begin{tabular}{|l|l|}
\hline NtaFe-SOD & Forward: CCCAGCATTCAACAATGCAGCTC \\
\hline Gene & Reverse: TCCGTTGGGCTTCATGGACTTC \\
\hline EF-1 $\alpha$ & Primers ('-3') \\
\hline & Forward: TGAGATGCACCACGAAGCTC \\
\hline L25 & Reverse: CCAACATTGTCACCAGGAAGTG \\
\hline & Forward: CCCCTCACCACAGAGTCTGC \\
\hline Poly(T) adapter & Reverse: AAGGGTGTTGTTGTCCTCAATCTT \\
& $\begin{array}{l}\text { Forward: } \\
\text { GCGAGCACAGAATTAATACGACTCACTATAGGTTTTTTTTTTTTAGCAT }\end{array}$ \\
\hline & Reverse: GCGAGCACAGAATTAATACGAC \\
\hline NtamiR160 & Forward: TGCCTGGCTCCCTGTATGCCA \\
\hline NtamiR164ab & Forward: AGAAGCAGGGCACGTGCA \\
\hline NtamiR164c & Forward: TGGAGAAGCAGGGCACATGCT \\
\hline Nta5.8S rRNA & Forward: ACGTCTGCCTGGGTGTCACA \\
\hline CrPR-1 & Forward: TAGTCCGAGCCAGAAAATGG \\
\hline & Reverse: ACGCTTTTACCGCATCACTC \\
\hline Cr-PR-5 & Forward: TACTCCTCCAGCAACCCTAGC \\
\hline \multicolumn{2}{l}{ Reverse: CGCCGTTAACTTCAACAATC } \\
\hline CrRSP9 & Forward: GATGGTGCACGTTTCCTTTT \\
\hline Primers for VIGS & Reverse: TGGGTCCTTCTCATCCAAAG \\
\hline Cr-IPR & Forward: CGGGATTCTCCAAGGAGATGGAGGTG \\
\hline & Reverse: CGGTCTAGAATAATCTGGATTGGCTTGAA \\
\hline Cr-IPR-(C)-F2 & Forward: GTGTTCAAGGCGTTT GTCC \\
\hline & Reverse: ACCGAAATGGACCAACTTG \\
\hline
\end{tabular}

${ }^{\mathrm{a}}$ : The nucleotides in italic and bold indicate the introduced restrictive cleavage site BamHI

b: The nucleotides in italic and bold indicate the introduced restrictive cleavage sites SpeI-SacI

${ }^{c}$ : The nucleotides in italic and bold indicated the introduced restrictive cleavage site SpeI

d: The nucleotides in italic and bold indicated the introduced restrictive cleavage site SacI 
Transgenic expression of Hyp-1 gene from Hypericum perforatum L. alters expression of defense related genes and modulates tolerance to Agrobacterium tumefaciens

Weina Hou ${ }^{1}$, Rupesh Kumar Singh ${ }^{2}$, Pan Zhao ${ }^{3}$, Viviana Martins ${ }^{4}$, Emmanuel Aguilar ${ }^{5}$, Tomás Canto ${ }^{5}$, Francisco Tenllado ${ }^{5 *}$, Alberto Carlos Pires $\operatorname{Dias}^{1,4,6}$

Supplemental Data

Table S2 Putative functional classification of transcripts identified by BLASTX analysis from two forward subtractive cDNA libraries from Agrobacterium tumefaciens-treated Hypericum perforatum L. suspension cells (12hr and 24hr)

\begin{tabular}{|l|l|l|l|l|l|l|}
\hline Clone No & Time & Hits & Accession & Description & E value & Origin \\
\hline 13,14 & $12 \mathrm{~h}$ & PLN02378 & PLN02378 & $\begin{array}{l}\text { Glutathione S-transferase } \\
\text { DHAR1 6.43e-90 } \\
\text { exogenous toxins) }\end{array}$ & Corchorus capsularis \\
\hline 91 & $12 \mathrm{~h}$ & PLN02906 & PLN02906 & $\begin{array}{l}\text { Xanthine dehydrogenase } \\
\text { (disease resistance) }\end{array}$ & $1.08 \mathrm{e}-62$ & Vicia caroliniana \\
\hline $\mathbf{9 4}$ & $\mathbf{1 2 h}$ & Bet_v1-like & $\mathbf{c d 0 7 8 1 6}$ & Hyp-1 (disease resistance) & $1.22 \mathrm{e}-09$ & Hypericum maculatum \\
\hline 168 & $12 \mathrm{~h}$ & PLN02243 & PLN02243 & $\begin{array}{l}\text { S-adenosylmethionine } \\
\text { synthase/polygalacturonase- } \\
\text { inhibiting protein (Pathogen } \\
\text { responsive) }\end{array}$ & $2.65 \mathrm{e}-07$ & \\
\hline
\end{tabular}




\begin{tabular}{|c|c|c|c|c|c|c|}
\hline Clone No & Time & Hits & Accession & Description & E value & Origin \\
\hline 13 & $24 \mathrm{~h}$ & $\begin{array}{l}\text { xylanase_inhib } \\
\text { itor_I_lik } \\
\text { TAXi_N }\end{array}$ & pfam14543 & $\begin{array}{l}\text { TAXI-I inhibits degradation } \\
\text { of xylan in the cell wall } \\
\text { (pathogen responsive) }\end{array}$ & $2.63 e-30$ & Gossypium raimondii \\
\hline $\begin{array}{l}8,11,92, \\
143,158\end{array}$ & $24 \mathrm{~h}$ & Bet_v1-like & cd07816 & Hyp-1 (PR-10) & $1.36 \mathrm{e}-123$ & Hypericum maculatum \\
\hline 13 & $24 \mathrm{~h}$ & GST_C_Tau & $\operatorname{cd} 03185$ & $\begin{array}{l}\text { GST_N family, Glutathione } \\
\text { S-transferase } \\
\text { responsive) }\end{array}$ & $1.59 \mathrm{e}-11$ & Manihot esculenta \\
\hline 16 & $24 \mathrm{~h}$ & PLN02582 & pfam13292 & $\begin{array}{l}\text { 1-deoxy-D-xylulose-5- } \\
\text { phosphate synthase } \\
\text { (Pathogen responsive) }\end{array}$ & $2.61 \mathrm{e}-75$ & Jatropa curcus \\
\hline 17 & $24 \mathrm{~h}$ & PLN00113 & PLN00113 & $\begin{array}{lr}\text { Leucine-rich } & \text { repeat (LRR) } \\
\text { protein } & \text { (Pathogen } \\
\text { Responsive) } & \end{array}$ & $1.60 \mathrm{e}-04$ & Populus euphratica \\
\hline 19 & $24 \mathrm{~h}$ & PLN02243 & PLN02243 & $\begin{array}{l}\text { S-adenosylmethionine } \\
\text { synthase } \quad \text { (Pathogen } \\
\text { Responsive) }\end{array}$ & $1.36 \mathrm{e}-123$ & Noccaea caerulescens] \\
\hline 72 & $24 \mathrm{~h}$ & SPFH_like_u4 & cd03407 & $\begin{array}{l}\text { SPFH (Stomatin, prohibitin, } \\
\begin{array}{l}\text { flotillin, and } \\
\text { suplK/C) }\end{array} \\
\begin{array}{ll}\text { responsive) } & \text { (Pathogen }\end{array}\end{array}$ & $2.34 \mathrm{e}-16$ & Citrus sinensis \\
\hline
\end{tabular}

